\title{
Acoustic Ducting, Reflection, Refraction, and Dispersion by Curved Nonlinear Internal Waves in Shallow Water
}

\author{
James F. Lynch, Fellow, IEEE, Ying-Tsong Lin, Member, IEEE, Timothy F. Duda, Senior Member, IEEE, and
} Arthur E. Newhall, Member, IEEE

\begin{abstract}
Nonlinear internal waves in shallow water have been shown to be effective ducts of acoustic energy, through theory, numerical modeling, and experiment. To date, most work on such ducting has concentrated on rectilinear internal wave ducts or those with very slight curvature. In this paper, we examine the acoustic effects of significant curvature of these internal waves. (By significant curvature, we mean lateral deviation of the internal wave duct by more than half the spacing between internal waves over an acoustic path, giving a transition from ducting to antiducting.) We develop basic analytical models of these effects, employ fully 3-D numerical models of sound propagation and scattering, and examine simultaneous acoustical and oceanographic data from the 2006 Shallow Water Experiment (SW06). It will be seen that the effects of curvature should be evident in the mode amplitudes and arrival angles, and that observations are consistent with curvature, though with some possible ambiguity with other scattering mechanisms.
\end{abstract}

Index Terms-Horizontal acoustic ducting, horizontal acoustic refraction, reflection, and dispersion, horizontal acoustic shadowing, shallow-water acoustics, 3-D acoustics, curved nonlinear internal waves.

\section{INTRODUCTION}

$\mathbf{N}$ ONLINEAR internal waves are common in the shallow waters of continental shelves and in adjacent seas, and have been shown to have strong effects on acoustic propagation and scattering. An early study in the Yellow Sea [1] showed a strong absorption of low-frequency $(50-1000 \mathrm{~Hz})$ sound due to scattering by internal wave packets, and this work was the impetus for many later studies. In the 1995 Shallow Water Acoustics in a Random Medium (SWARM) experiment [2], strong mode coupling occurring with across-internal-wave propagation geometry was emphasized, with the main acoustic tracks being directed across shelf. However, also as part of SWARM, Badiey et al. [3] performed transmissions in an along-shelf (and thus along the nonlinear internal

Manuscript received May 14, 2009; revised December 01, 2009; accepted December 04, 2009. Current version published February 10, 2010. This work was supported by E. Livingston and T. Pawluskiewicz of the U.S. Office of Naval Research (ONR) under Grant N00014-04-1-0146 and the ONR postdoctoral fellowship award Grant N00014-08-1-0204.

Associate Editor: N. R. Chapman.

The authors are with the Applied Ocean Physics and Engineering Department, Woods Hole Oceanographic Institution, Woods Hole, MA 02543 USA (e-mail: anewhall@whoi.edu).

Color versions of one or more of the figures in this paper are available online at http://ieeexplore.ieee.org.

Digital Object Identifier 10.1109/JOE.2009.2038512 wavefronts) geometry. They observed a very strong ducting of low-frequency (roughly $50-500 \mathrm{~Hz}$ ) sound between nonlinear internal waves. This effect had been predicted by computer modeling [4] and normal mode theory [5], but not previously observed. A strong and acoustically important ducting effect of order $6-10 \mathrm{~dB}$ was observed. The ducting and lateral mode scattering due to curved internal waves, previously examined for straight internal waves, will be the main topics of this paper.

In this paper, the acoustic effects of curved internal waves will be examined using theory, numerical modeling, and experimental data. Specifically, we will be looking at: ducting of sound through curved internal wave ducts, dispersive refraction of sound out of such curved ducts, and reflection and refraction of energy for sound sources external to such ducts. To do this, we will provide some basic analytic expressions which can readily show the physics, dependence on system parameters, and propagation effects. These expressions also allow "back of the envelope" estimates of acoustic field effects. With this foundation in place, results from more complicated calculations can be more systematically examined, namely, from fully three-dimensional (3-D) numerical simulation of propagation in and through curved internal waves. Finally, we will look at data from the 2006 Shallow Water Experiment (SW06) [6], which can shed some light on the topics we discuss here. SW06 was not specifically designed to address these issues and does not provide a definitive data set. However, features in the arrival angles and intensities consistent with the effects considered here appear in the data.

Our paper is organized as follows. Following this Introduction, we present in Section II our measurements of wavefront curvature from SW06, taking this to be a "not atypical" continental shelf environment for nonlinear internal waves. In Section III, we present some basic theory for how low-frequency acoustic ducting, reflection, refraction, and dispersion by curved nonlinear internal waves works, using the Weinberg-Burridge [7] formalism as a framework. In Section IV, we present computer modeling results, which give a visual and easy to comprehend depiction of the physical processes. In Section V, we present SW06 data showing just how large this effect is for one particular geometry, frequency, and set of internal wave field realizations using arrival angle fluctuations as the variable of interest. In Section VI, we present our conclusions and recommendations for future work. 


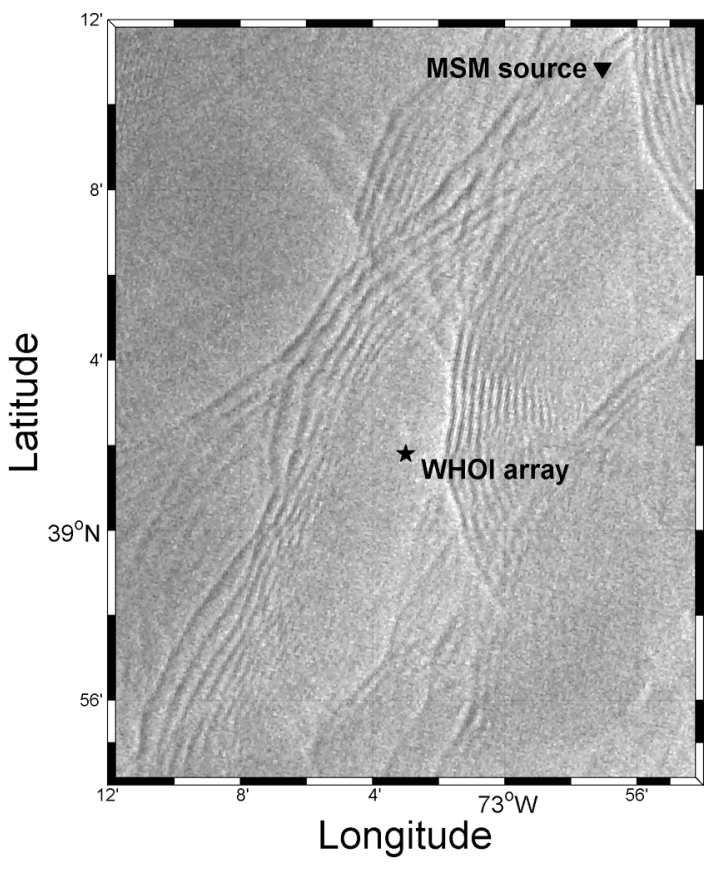

Fig. 1. SAR image of curved nonlinear internal waves in the SW06 experimental area. Range from the University of Miami acoustic source (MSM) to the Woods Hole Oceanographic Institution (WHOI) array is $19.7 \mathrm{~km}$, and orientation is along shelf.

\section{Measurements of Internal Wave CuRvature}

To date, most of the acoustic propagation studies of internal waves have used straight line internal wavefronts for individual waves or packets. (Notable exceptions are the works by Katznelson and Pereselkov [5] and Duda et al. [8].) However, while nearly linear wavefronts have been observed and make sense due to generation at linear shelfbreaks, reasonably curved internal wavefronts are just as commonly observed. This is illustrated using satellite synthetic aperture radar (SAR) images. Fig. 1 shows typical curved wavefronts from a SAR image from SW06. A curved wavefront can arise from wave generation at a strong bathymetric "point feature" (compared to the internal tide wavelength of $\sim 10-40 \mathrm{~km}$ ) such as a submarine canyon like Hudson Canyon, or it can be produced by horizontally varying bathymetry or by flow features (current shear and/or buoyancy frequency variations) refracting an initially plane wave.

Curved wavefronts can be modeled as circular arcs, and that technique is used in this paper, which is focused on understanding the physical effects on acoustics of curved, nonlinear internal waves, rather than providing a detailed numerical/oceanographic model of such waves. We will take the local wavefront curvature to be consistent with field observations, both from SAR and in situ observations (see [9] for in situ data discussions).

When looking at the SAR images of nonlinear internal wave surface effects, one can employ image processing schemes of varying degrees of complexity. As an example, to identify and map the waves on the surface, Boughan and Siegmann [10] have used fingerprint recognition methodology with reasonable success. Gradient sensing (edge detection) algorithms also have some success in this mapping task (e.g., standard commercial

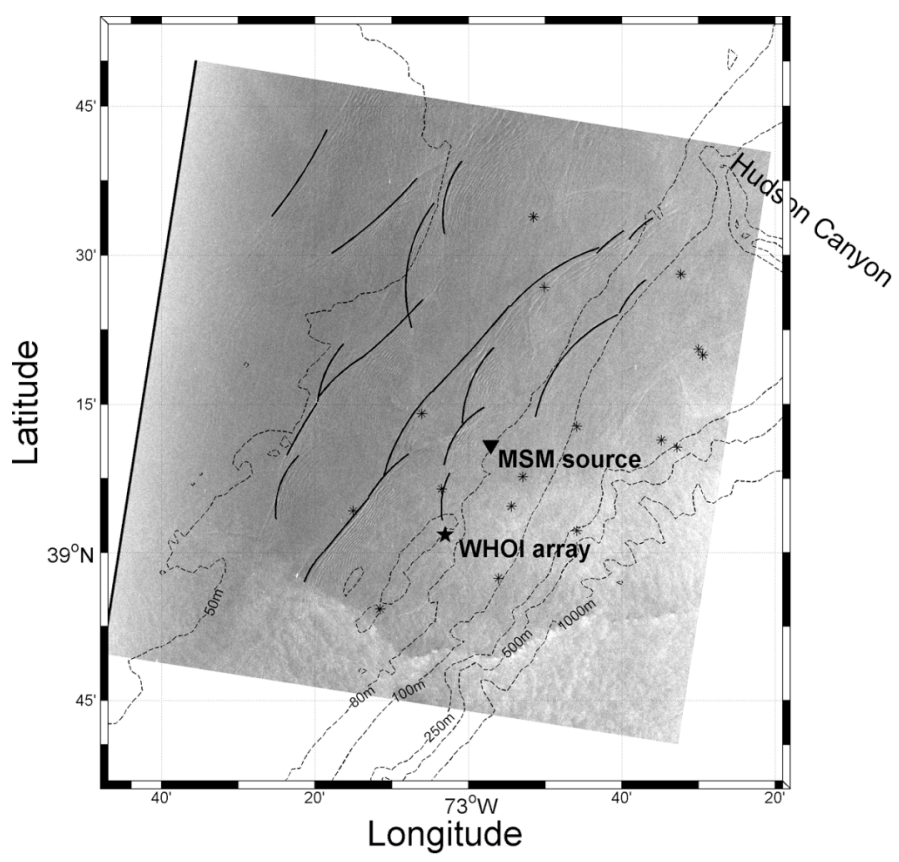

Fig. 2. Curved internal wave train significant wavefronts seen in SW06, from one SAR image (i.e., a "realization"). Fitted circular arcs are shown. Center points of the fitted circles are also shown $(*)$.

signal processing software). However, the most sensitive and discriminating sensor to map these waves may be the trained human eye, which detects low signal-to-noise ratio (SNR) signals well and also recognizes patterns versus clutter better. This point can certainly be argued, but we will take the pragmatic view that for the small number of images that we have available to process (order 10), visual processing on image features is both robust and economical. This approach also can be argued to be subjective - to minimize this, we had several independent observers cross check the imaging results for consistency.

The results of fitting circular curves to prominent and/or significant internal waves in a typical SAR image from SW06 are shown in Fig. 2. In this figure, a number of arcs of various lengths and radii of curvature can be seen. Three things are of first-order interest to us in this picture: 1) the distribution of arc lengths, 2) the distribution of radii of curvature, and 3) the seemingly spatially random distribution of the circle centers. The distribution of radii of curvature, which is most important to this paper as input to the acoustics, shows the forms seen in Fig. 3(a) and (b). We see that these distributions of radii peak at $\sim 20-25 \mathrm{~km}$, which intriguingly is the along-shelf correlation scale for the density and current field, and may indicate some of the underlying mechanisms for the curvature (as will be discussed). We note that the distribution for the leading waves of packets [Fig. 3(b)] tends towards a somewhat larger radius of curvature than a more inclusive distribution [Fig. 3(a)], which may reflect the nonlinear "healing or conjoining" of intersecting and nonlinearly interacting waves, as well as the simple "distance from the source" effect. The distribution of arc lengths [Fig. 3(c)] is similarly peaked at $\sim 20-25 \mathrm{~km}$, which tells us how long the ducted acoustic track can be. The spatial distribution of where the centers of the arc lie should eventually tell us about the source distribution for the curvature. As mentioned before, 

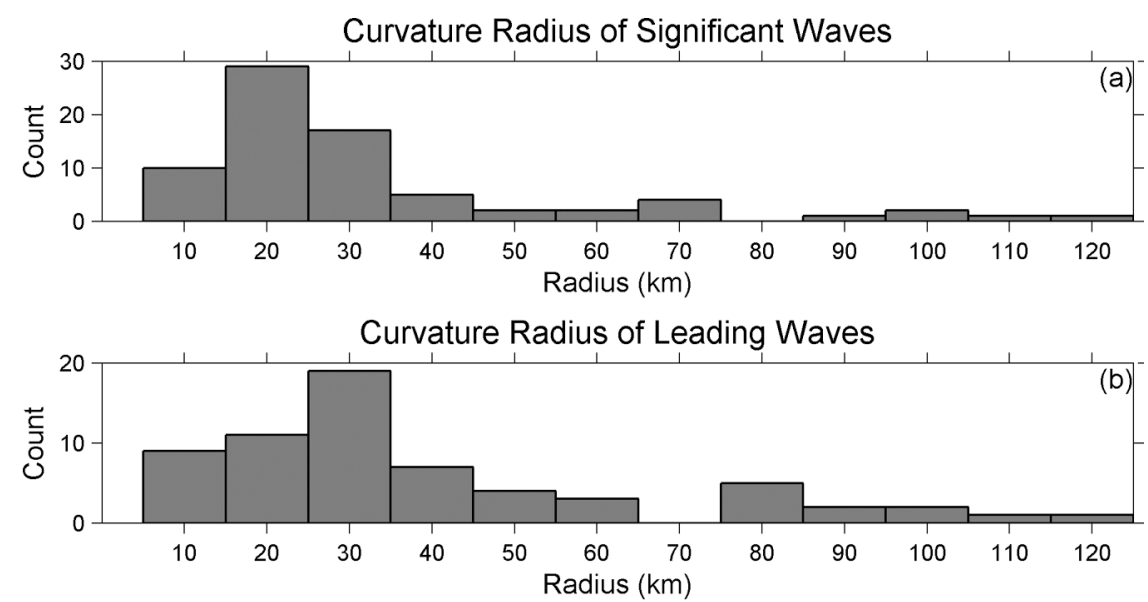

Arc Length of Leading Waves

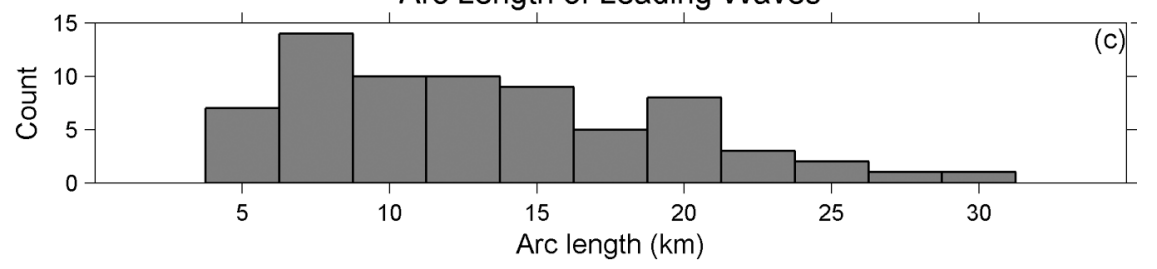

Fig. 3. (a) and (b) Distribution of radii of curvature from a SAR image, like the one shown in Fig. 2. (c) Arc lengths of the leading waves.

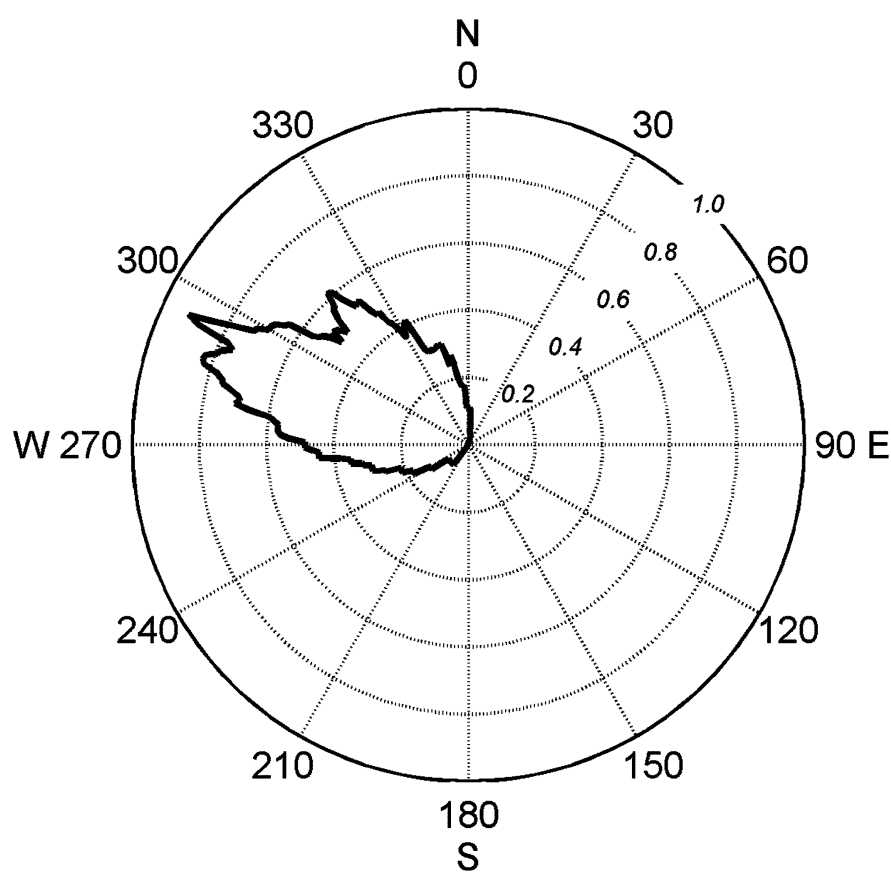

Fig. 4. Curved internal wavefront directionality from SAR images.

this can be due to several effects, and will be looked at in the near future.

Another quantity of interest is the directional spectrum of the nonlinear internal waves, shown in Fig. 4. This figure shows that the waves travel primarily in the $290^{\circ}$ direction relative to true north, but also have a spread of about $30^{\circ}$ to either side. This is due to both the curvature and the primary direction of the wave trains. This spread also indicates the probability of a unidirectional, along-shelf acoustic track encountering the internal waves at an angle, which can lead to mode coupling effects.
Although the radii of curvature, arc lengths, and somewhat scattered locations of the curvature centers given by the SAR images provide sufficient information for a theoretical acoustics study, there is also the aspect of predicting these numbers from physical oceanographic models. Currently, primitive-equation hydrostatic regional ocean models work routinely at space and time scales of a few kilometers $x-y$ resolution and 12.4 h (M2 tide) time scales, but not at the 10-m and 30-s time scales needed for nonlinear internal waves with nonhydrostatic dynamics. Three-dimensional high-resolution nonhydrostatic models have been developed but are not in routine use for internal wave studies [11], [12]. A "nested internal wave model" superimposed on a mesoscale model is currently close to the state-of-the-art and feasible to do. However, if refraction by mesoscale eddies and generation of curved waves by irregular features (e.g., canyons) near the shelfbreak are the prime sources of the curved internal waves we see, then we can perhaps predict the curvature quantities presented above simply with mesoscale oceanography models, reasonable bathymetry maps, and relatively simple refraction/scattering physics. This approach is currently being pursued [13].

\section{THEORETICAL STUDY}

In this section, we wish to provide some analytic expressions which show the physics of the ducting, reflection, refraction, and dispersion of sound by curved nonlinear internal waves. Specifically, the derived expressions show the dependence on sonar system and environmental parameters, and predict intensity and arrival angle variations. We start from the Weinberg-Burridge "horizontal rays and vertical modes" picture which can accommodate 3-D adiabatic modes and even some coupled mode effects, when used properly.

A side view of the environment to be used with this model is shown in Fig. 5. Far away from the internal waves there is an 


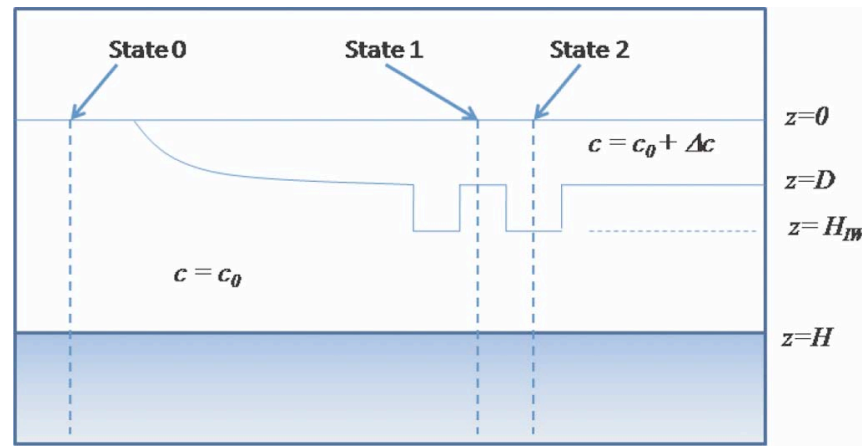

Fig. 5. Background state (state 0 ) and two perturbation states.

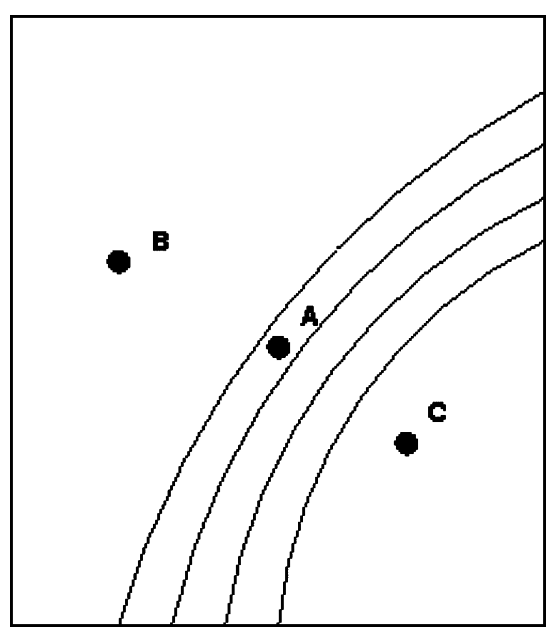

Fig. 6. Useful acoustic source geometries to consider relative to a curved internal wave train.

isospeed background state with sound speed $c_{0}$, depth $H$, and with modal wave numbers $k_{0 n}$. Between two internal waves is perturbation state 1, where a near-surface upper layer of height $D$ having higher sound speed exists, with upper layer speed $c_{0}+$ $\Delta c$. In this region, the mode wave numbers are denoted $k_{1 n}$. Inside the internal wave is found perturbation state 2 , where the upper layer of elevated sound speed extends to depth $H_{I W}$ and mode wave numbers are denoted $k_{2 n}$. Note that a duct forms between internal waves (state 1 bounded by state 2 on either side).

Fig. 6 is a plan view of possible source positions relative to a curved (circular) internal wave train. There are three distinct geometries to consider: A) a source within the internal wave train, B) a source on the exterior of the circular wavefronts, and C) a source interior to the internal wavefronts. We consider each of these cases.

\section{A. Critical Angle Estimation}

In this section, an expression for the critical angle within an internal wave duct will be derived in terms of the internal wave parameters. Local eigenvalues can be computed numerically and used to study ducting directly, but the goal here is to show how the internal-wave parameters govern the ducting process. Perturbation theory will be used to derive an expression governing the total internal reflection within internal wave ducts. Internal wave ducts will be approximated as square waves, for theoretical convenience. However, this simplification still represents the ducting effects of the waves well.

The chosen Weinberg-Burridge (or "ray mode" for shorthand) approach is initiated by calculating the local vertical mode eigenvalues for the 3-D volume to be considered on a well sampled $x-y$ grid, producing $k_{n}(x, y, \omega)$ for a given source angular frequency $\omega$. One then interpolates the eigenvalues on this $x-y$ grid for each mode (at the given $\omega$ ). Doing this, one creates a horizontal index of refraction field that will determine the horizontal trajectories for each vertical mode $n$, using the simple relation $n_{n}(x, y, \omega)=k_{n}(\vec{r}) / k_{n}(0)$. Here we choose the reference point (origin) to be the point $\vec{r}=(x, y)$ with the smallest eigenvalue magnitude, so that $n_{n} \geq 1$.

Snell's law, written in terms of grazing angle with respect to the horizontal boundary of an internal wave duct, is $k_{1 n} \cos \theta_{1}=$ $k_{2 n} \cos \theta_{2}$ for vertical mode number $n$. Disregarding tunneling effects [14], the critical angle for trapping a mode within an internal wave duct is given by

$$
\theta_{1 n}^{\text {crit }}=\cos ^{-1}\left(\frac{k_{2 n}}{k_{1 n}}\right)
$$

To complete the task of expressing the critical angle in terms of the parameters of depth, internal wave height, and so on, we use perturbation theory. Since we are mainly interested in modeling oceanographic effects due to internal waves, we can take the bottom to be a rigid $(\rho \rightarrow \infty)$ boundary for simplicity. (That is not to say that the bottom properties do not affect this problem - they do [15], [16]. However, we will ignore that added complication here.)

Away from the internal waves, the rigid bottom model background-state eigenvalues are found via

$$
\gamma_{0 n} H=\left(n-\frac{1}{2}\right) \pi
$$

and

$$
k_{0 n}=\sqrt{k^{2}-\gamma_{0 n}^{2}}
$$

where $\gamma_{0 n}$ is the vertical mode eigenvalue/wave number and $k=\omega / c_{0}$. The vertical mode functions for this model are sinusoids, i.e.,

$$
Z_{0 n}(z)=\left[\frac{2}{H}\right]^{1 / 2} \sin \left(\gamma_{0 n} z\right) .
$$

It is the difference between these sinusoids and the mode functions for a more realistic stratified ocean and seabed that actually represent the biggest limitation for our model. In a more realistic ocean, the low modes are pushed down from the mixed layer and the internal waves more than this background model permits. However, this affects the excitation of the modes far more than their horizontal trapping, so it is not a major concern in our treatment here.

To treat the mixed layer (state 1) and internal wave (state 2) situations, we use the well-known perturbation forms [17] to find the mode wave numbers

$$
k_{1 n}=k_{0 n}+\Delta k_{1 n}, \quad k_{2 n}=k_{0 n}+\Delta k_{2 n}
$$


namely, for state 1

$$
\begin{aligned}
\Delta k_{1 n} & =\frac{1}{k_{0 n}} \int_{0}^{H} \frac{\Delta q Z_{0 n}^{2}(z) d z}{\rho(z)} \\
\Delta q & =-\Delta c(z) \frac{\omega^{2}}{c_{0}^{3}(z)}
\end{aligned}
$$

where $\omega$ is angular frequency. In (4a) and (4b), $c_{0}(z)$ is the background sound-speed profile (which is isospeed in this case). State 2 produces similar expressions.

Inserting the mode shapes into (4a) and noting that the integration range can be changed because $\Delta c$ is zero in the lower layer, one can write

$$
\Delta k_{1 n}=\frac{-2}{k_{0 n} H} \frac{\omega^{2}}{c_{0}^{2}} \frac{\Delta c}{c_{0}} \int_{0}^{D} \sin ^{2}\left(\gamma_{0 n} z\right) d z .
$$

The sine squared integral is an elementary one, giving (denoting the integral by $I$ )

$$
\begin{aligned}
I & =\left[\frac{z}{2}-\frac{1}{4 \gamma_{0 n}} \sin \left(2 \gamma_{0 n} z\right)\right]_{z=0}^{z=D} \\
& =\frac{D}{2}\left(1-\operatorname{sinc}\left(\frac{2 \gamma_{0 n} D}{\pi}\right)\right)
\end{aligned}
$$

where the sinc function is defined as $\operatorname{sinc}(X)=\sin (\pi X) / \pi X$. In the high-mode limit, as $\gamma_{0 n}$ increases and so does the argument of the sinc function, the second term becomes small with respect to the first, giving the form

$$
I \approx \frac{D}{2} .
$$

As an example of where the high-mode number approximation is valid, when the value of the sinc function in (6) is less than $\sim 0.2$, we can neglect its contribution to the integral $I$. This criterion can be shown to be equivalent to $5 n>H / D$, where $n$ is the mode number. In this level of approximation, the eigenvalue perturbations are

$$
\Delta k_{1 n}=\frac{-1}{k_{0 n}} \frac{\omega^{2}}{c_{0}^{2}} \frac{\Delta c}{c_{0}} \frac{D}{H}
$$

A procedure identical to that used to derive (8) can be used to find the eigenvalue perturbations for state 2 , in the internal wave of depression

$$
\Delta k_{2 n}=\frac{-1}{k_{0 n}} \frac{\omega^{2}}{c_{0}^{2}} \frac{\Delta c}{c_{0}} \frac{H_{I W}}{H} .
$$

Note that although the exact form of the integral $I$ is generally a better approximation to use, the high-mode number approximation shows very clearly the mode number, frequency, layer thickness, and sound-speed perturbation contribution to the wave number perturbation and thus the internal wave induced index of refraction change in the adiabatic mode model.

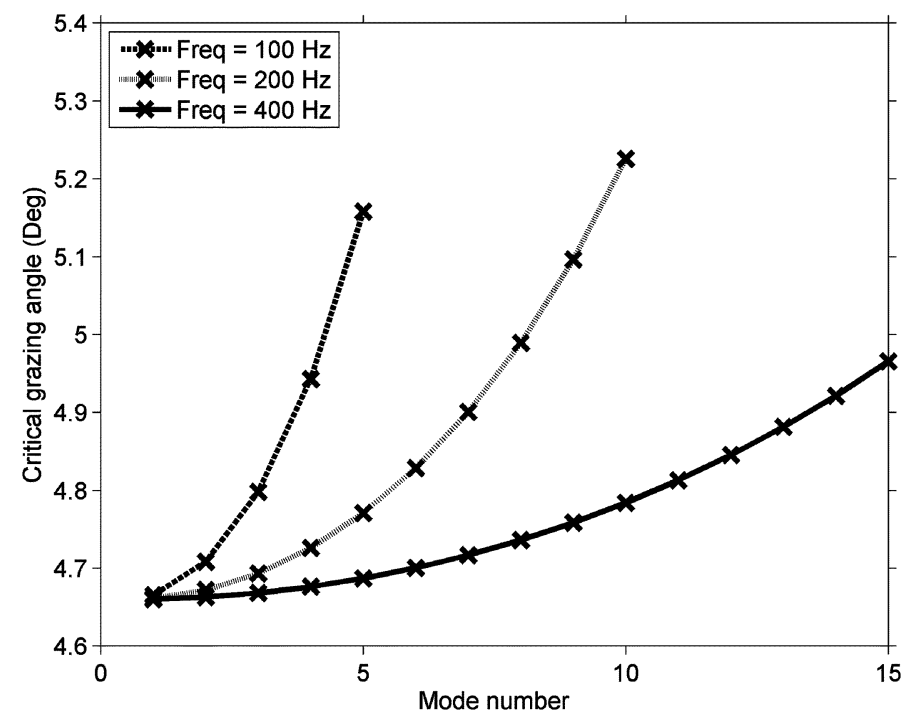

Fig. 7. Critical grazing angle versus mode number and frequency for an idealized internal wave based on the real SW06 internal waves.

Combining (1), (2a), (3), (8), and (9) yields for critical grazing angles

$$
\theta_{1 n}^{\text {crit }}=\cos ^{-1}\left[\frac{\left[k^{2}-\left[\frac{\left(n-\frac{1}{2}\right) \pi}{H}\right]^{2}\right]^{1 / 2}-\frac{H_{I W} \Delta c \omega^{2}}{k_{0 n} c_{0}^{3} H}}{\left.\left[k^{2}-\left[\frac{\left(n-\frac{1}{2}\right) \pi}{H}\right]^{2}\right]^{1 / 2}-\frac{D \Delta c \omega^{2}}{k_{0 n} c_{0}^{3} H}\right] .}\right.
$$

The above formula shows the limit of no trapping by internal waves (critical grazing angle of $0^{\circ}$ ), when $H_{I W}=D$. When these two dimensions differ, modes having supercritical incidence angle will be trapped. Modes with subcritical incidence angle will dispersively refract as they exit the duct.

The equation for $\theta_{1 n}^{\text {crit }}$ can be evaluated for waveguide parameters based on SW06 data. We take $D=15 \mathrm{~m}, H_{I W}=25 \mathrm{~m}$, $H=80 \mathrm{~m}, c_{0}=1520 \mathrm{~m} / \mathrm{s}, \Delta c=40 \mathrm{~m} / \mathrm{s}, f=100,200$, and $400 \mathrm{~Hz}$, which results in the critical angle curves seen in Fig. 7. In this figure, a number of interesting features can be observed. First, one sees that the critical angles range from about $4.65^{\circ}$ to $5.2^{\circ}$, with mode 1 for all frequencies being at the minimum of $4.65^{\circ}$. Second, we see that the curves are all parabolae, in accordance with (10). Third, we see that as frequency increases, the curves flatten, and will reach a high-frequency asymptote of $4.65^{\circ}$, which is the ray theory limit. Calculations using real SW06 internal wave and waveguide environmental parameters give critical angles close to those seen in Fig. 7, except for some minor differences due to the penetrable bottom.

\section{B. A Source Within the Internal Wave Train}

Having created a useful, horizontal index of refraction field $n_{n}(\vec{r})$ for each mode, we can now turn our attention to the hori- 


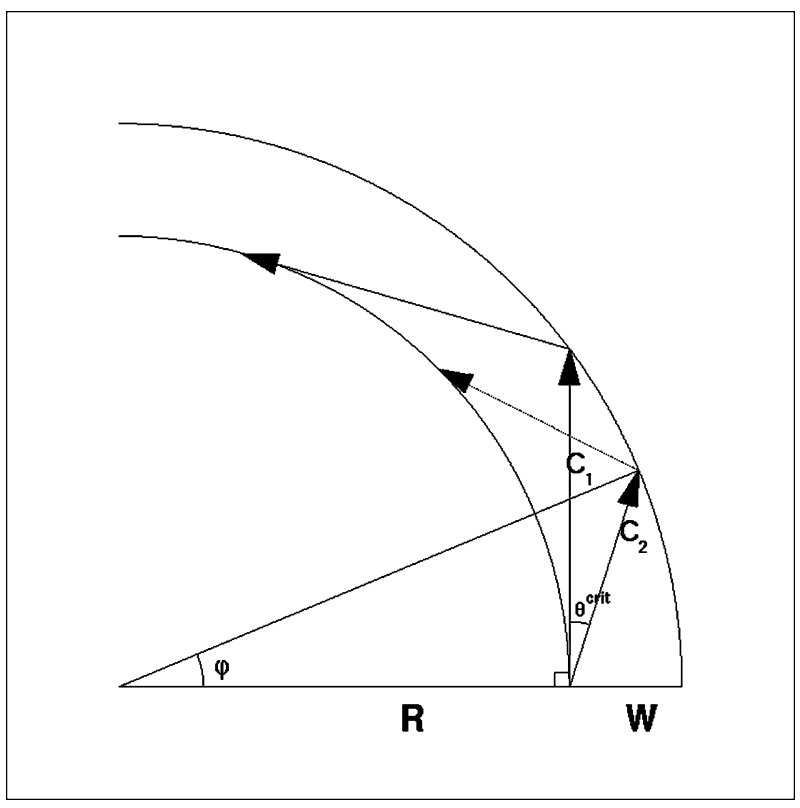

Fig. 8. Geometry of a low-speed duct between two internal waves. Limiting chords for modal-ray propagation are shown.

zontal propagation and scattering effects that are the main focus of the paper.

The point of this section is to determine limitations on curved wave duct width $(W)$ and radius of curvature $(R)$ which must be satisfied for two types of ducting. One type is full ducting, where horizontal modes of the duct interact strongly with both internal wave "walls," and the other is equivalent to repeated reflection on the exterior (concave) wall. These would be called potential well and whispering gallery effects in physics parlance.

Total internal reflection and leakage of modes in a curved waveguide have been studied extensively in terms of optics (light pipes) and microwave ducts, among other fields [18]. Our treatment here will obviously bear some similarities to these analogous physical systems, as well as some differences due to the specific nature of the ocean acoustic waveguide. To understand the basic physics of a sound source within an internal wave train, consider a single duct, which might be formed by, e.g., the leading edge or strongest wave of an internal wave train and its nearest neighbor. A source in a canonical horizontal duct of this type, where the square-well internal wave approximation is used, is shown in Fig. 8. This figure defines a few parameters that are needed for the analysis. $C_{1}$ is the length of a line segment (ray) that is tangent to the inner wall and reflects off the outer wall. $C_{2}$ is the length of a ray that reflects off the outer wall at a critical angle while being emitted at the inner wall at the same angle $\theta . \Delta_{n m}$ is the arc length of the mode-cycle distance for horizontal ducted mode $m$ of vertical mode $n . \varphi$ is the radian arc length associated with $\Delta_{n m} / 2$.

Since internal wave trains have across train extent $100-400 \mathrm{~m}$ for both the depression-type internal waves and the low-sound-speed (acoustic duct) regions between them, which is many acoustic wavelengths for frequencies above about 50 $\mathrm{Hz}$, it is reasonable to use the square-well approximation. In this approximation, one assumes infinite width of the bounding internal waves. This is allowable if tunneling is small. This method also treats refractive transmission correctly. Internal waves are also slowly varying in sound speed over their dimensions (except at boundaries, which we treat explicitly by transmission/reflection coefficients), so that our square-well model and horizontal ray theory are also consistent.

The first effect of note due to the curvature of the waveguide is on the angle of reflection off the boundaries of the waveguide. For an infinitely thin waveguide, $W \rightarrow 0$ and there is no local curvature effect, as can be seen examining Fig. 8. However, for finite $W$, the waveguide exhibits a curve over a horizontal ray/mode cycle distance $\Delta_{n m}$, where $n$ is the vertical mode index, and $m$ is the horizontal mode index. Note that the horizontal mode cycle distance is defined by the interference of two adjacent horizontal modes ( $m$ and $m+1)$. The arc length (in radians) over half a cycle distance (the distance from hitting one wall and going to the opposite one) can be estimated with midpoint and small-curvature approximations, and is found to be

$$
\varphi_{n m}=\tan ^{-1}\left(\frac{\frac{\Delta_{n m}}{2}}{R+\frac{W}{2}}\right) .
$$

$\Delta_{n m}$ can be computed from knowledge of the (straight waveguide) horizontal modes via $\Delta_{n m}=2 W / \tan \theta_{n m}^{\text {hor }}$, which relates $\Delta_{n m}$ directly to the horizontal mode angle $\theta_{n m}^{\text {hor }}$. This mode angle can be computed for our model using an eigenvalue equation directly analogous to the Pekeris waveguide equation. However, we can replace the $\Delta_{n m}$ calculation by its bounding limits by considering chords at $C_{1}$ and $C_{2}$. These represent the cycle distance limits for the horizontal modes formed from the sound energy bouncing between the internal wave walls ("bouncing" modes). Note that there should also be horizontal modes of a true "whispering gallery" type (i.e., only reflecting off the exterior wall), as will be discussed. The results of using these limits are shown in

$$
\begin{aligned}
C_{1}= & \sqrt{(R+W)^{2}-R^{2}} \\
C_{2}= & \frac{W+R}{\sin \left(\frac{\pi}{2}+\theta_{n}^{\text {crit }}\right)} \\
& \times \sin \left(\pi-\left(\frac{\pi}{2}+\theta_{n}^{\text {crit }}\right)-\sin ^{-1}\left(\frac{R \sin \left(\frac{\pi}{2}+\theta_{n}^{\text {crit }}\right)}{W+R}\right)\right) \\
= & \frac{W+R}{\cos \theta_{n}^{\text {crit }}} \cos \left(\theta_{n}^{\text {crit }}+\sin ^{-1}\left(\frac{R}{W+R} \cos \theta_{n}^{\text {crit }}\right)\right)
\end{aligned}
$$

and $C_{2} \leq \Delta_{n m} / 2 \leq C_{1}$, where $\theta_{n}^{\text {crit }}$ can be calculated from Snell's law, as discussed previously, and shown explicitly in (10). Substituting (12a) in (11) gives the limits for $\varphi$

$$
\tan ^{-1}\left[\frac{C_{2}}{R+\frac{W}{2}}\right] \leq \varphi \leq \tan ^{-1}\left[\frac{C_{1}}{R+\frac{W}{2}}\right]
$$

which is an explicit formulation for the limits of the angle of internal wave curvature over half a cycle distance of the bouncing horizontal modes. The detailed sound-speed profile and internal 
wave structures play a role in these limits because $C_{2}$ is a function of the critical angle. Also, it can be shown that for higher vertical modes the lower bound is smaller, which implies a better ducting condition.

Let us further discuss the transition of the ducting physics from straight internal waves to curved internal waves. First, we note that in some cases (the bouncing mode cases), averaged over a cycle distance, the modal energy will hit the interior wall of the curved duct at a lower angle $\theta_{n m}^{\text {hor }}-\varphi / 2$ than the exterior wall, which is at $\theta_{n m}^{\text {hor }}+\varphi / 2$, where $\theta_{n m}^{\text {hor }}$ is the horizontal mode angle obtained from a straight duct. This is a "falling away horizon" (interior wall) versus an "approaching horizon" (exterior wall) curvature effect. Thus, losses should occur preferentially at the exterior side of the curved duct. Moreover, all of the bouncing modes should have incoming grazing angles of $\theta_{n m}^{\text {crit }}-\varphi / 2$ or below at the exterior side of the curved duct. Sound that is subcritical and reflects at the interior wall of the duct may be supercritical on the outer wall of the wave duct because of this restriction. Some energy will escape via dispersive transmission, with the angle of escape depending on the mode numbers $(n$ and $m$ ) and the frequency $\omega$. This effect will be demonstrated later with a computational result. Also note that the forms described above can also be used to identify the energy carrying horizontal rays that such a 3-D parabolic acoustic wave equation (PE) produces.

In the whispering gallery mode situation, the modal energy will hit the exterior wall at $\theta_{n m}^{\text {hor }}+\varphi / 2$ on average over a cycle distance. From Fig. 8, it is clear that if this angle is smaller than the tangent angle $\cos ^{-1}(R /(R+W))$, at which the reflected sound from the outer wall will just graze the inner wall, one can see a horizontal mode/ray reflecting only from the outer boundary, which leads to the classic whispering gallery effect and its modes.

\section{A Source Exterior to Curved Internal Wave Train}

Whereas the case of a source within a curved internal wave duct can produce some interesting light pipe and chromatic dispersion effects [18], a source exterior to a curved internal wave train can see some equally interesting horizontal shadow zone and caustic effects. The shadow zone arises because the circular internal wavefront is not transparent to sound below critical grazing angle. The caustic is associated with the edge of the shadow zone.

To examine this case, consider the geometry depicted in Fig. 9. Here, $R$ is the radius of the circular internal wave train, $R_{S}$ is the distance from the source to a given point on the internal wavefront, and $R_{\mathrm{CPA}}$ is the closest point of approach (closest distance) from the source to the circular internal wave train. In terms of angles, $\theta_{0}$ is the horizontal launch angle of the sound relative to the axis between the original circle and the source, $\varphi$ is the angle between the radius to the point on the curve of interest and the source/circle origin axis, and $\theta^{\text {graz }}$ is the grazing angle of the sound with the internal wave train surface, the quantity of prime interest here. Application of the law of sines gives us

$$
\frac{R}{\sin \theta_{0}}=\frac{R+R_{\mathrm{CPA}}}{\sin \left(\frac{\pi}{2}+\theta^{\mathrm{graz}}\right)}=\frac{R_{S}}{\sin \varphi} .
$$

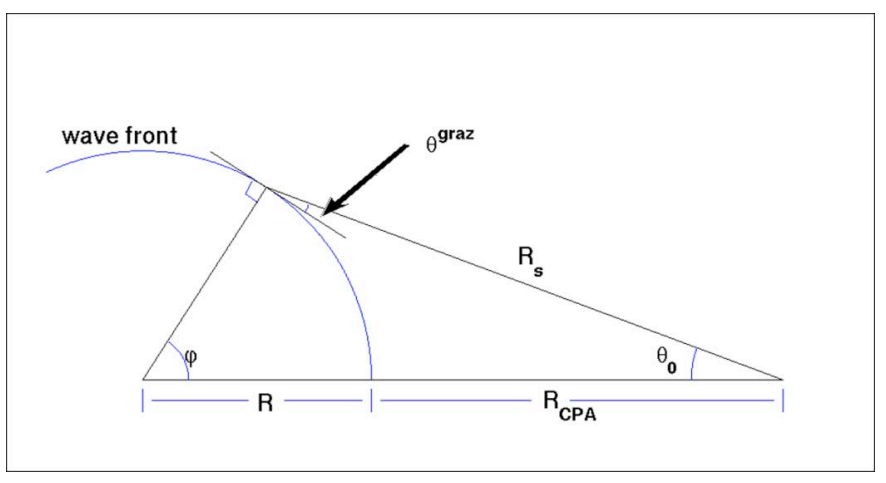

Fig. 9. Example of how "falling away horizon" gives critical angle before the tangent point, and thus a shadow region behind the internal wave.

This can be solved to give

$$
\theta^{\mathrm{graz}}=\cos ^{-1}\left[\frac{R+R_{\mathrm{CPA}}}{R} \sin \theta_{0}\right] .
$$

Past critical grazing angle out to the angles where the ray hits the tangent of the circle, there is an acoustic shadow. The latter (tangent) angle is given by

$$
\theta^{\text {tangent }}=\cos ^{-1}\left(\frac{R}{R+R_{\mathrm{CPA}}}\right) .
$$

There will be a shadow beyond the internal wave along extensions of the rays that strike the wave duct such that $0<\theta^{\text {graz }}<\theta^{\text {crit }}$. The interest here is not just that there is critical angle shadowing, which we would get with a straight line internal wavefront as well, but since the "horizon is falling away" due to the internal wavefront curvature, one sees a shadow at a much lower $\theta_{0}$ than one would for the straight line front case. This also reduces the distance that is required between the source and the internal wave for shadowing to occur, which means less energy lost in transit, making shadowing more important. Again, we note that $\theta^{\text {crit }}$ can easily be determined given the forms we have derived above.

\section{A Source Interior to Curved Internal Wave Train}

Looking at Fig. 10, we see that the law of sines and standard triangle identities can be used exactly as in Section VI, so as to give

$$
\frac{R}{\sin \left(\pi-\theta_{0}\right)}=\frac{R_{S}}{\sin \varphi}=\frac{R-R_{\mathrm{CPA}}}{\sin \left(\frac{\pi}{2}-\theta^{\text {graz }}\right)}
$$

Thus, we obtain

$$
\theta^{\mathrm{graz}}=\cos ^{-1}\left[\frac{R-R_{\mathrm{CPA}}}{R} \sin \theta_{0}\right] .
$$

Equation (18) is the same as (15), only with a sign change for $R_{\mathrm{CPA}}$ (which is now negative, as it is inside the circle).

In this case, there is a concave horizon (approaching) and it is difficult to attain critical grazing angle (impossible when $\left.\theta^{\text {graz }} \geq \theta^{\text {crit }}\right)$. Thus, sources inside the internal wave are less likely to see any critical angle shadowing effects. 


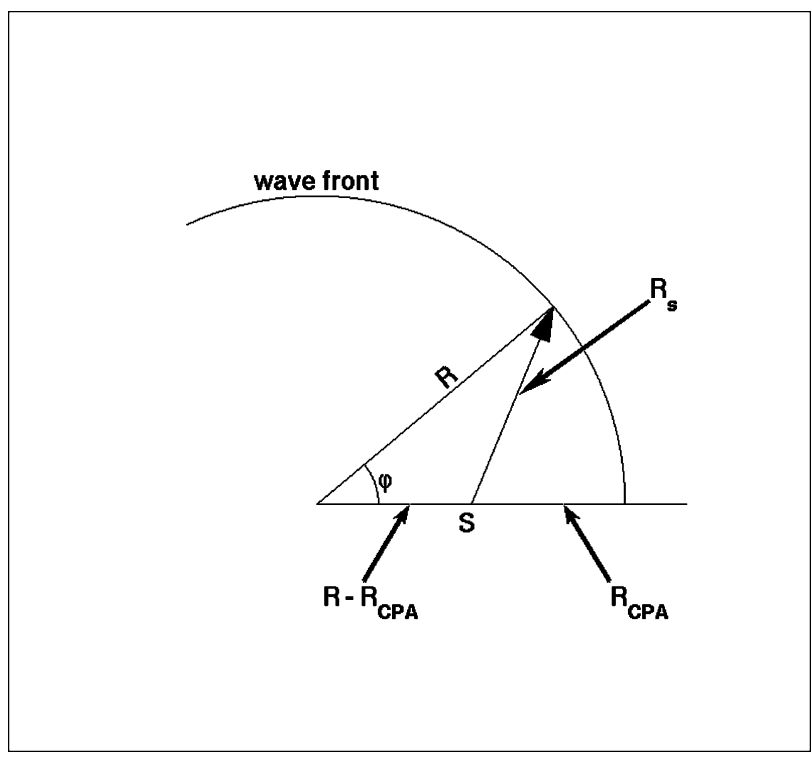

Fig. 10. Source interior to internal wave train.

\section{E. Energetics}

From our theoretical analysis, we can conclude that modes can both duct and be bent appreciably in the horizontal. But what do we expect to see in real data? To partially answer that question, we will look briefly at the energetics of the ducted and leaked beams using a sonar equation/geometric approach first used by Lynch et al. [19] in the context of ducting within straight internal waves. In the straight-duct picture, one finds that for $\theta<\theta^{\text {crit }}(\theta$ is the horizontal grazing angle), ducting occurs and there is no cylindrical spreading, i.e., the $r^{-1 / 2}$ cylindrical spreading term vanishes, and so one sees a ratio $R$ between the trapped (ducted) and horizontally untrapped (cylindrically spreading) pressures

$$
R_{\text {press }} \cong \sqrt{\frac{2 r \theta^{\text {crit }}}{W}}
$$

where $r$ is the source-to-receiver range and $W$ is the width of the duct. This sonar equation/geometry factor reasonably predicted what was seen in the 1995 SWARM experiment data [2]. We now extend this form to look at the ratio of the higher mode ducted energy to the lower mode unducted (beam-leaked) energy. If we look at our $100-\mathrm{Hz}$ example in Section III-A, this is the ratio of vertical mode 1 to mode 2. Note that in Section III-A a rigid bottom was used, while a penetrable bottom with sound speed $1700 \mathrm{~m} / \mathrm{s}$ and density $1.8 \mathrm{~g} / \mathrm{cm}^{3}$ is used here. If we write down expressions for the pressure of these modes, we have

$$
p_{1}(r)=\frac{1}{\sqrt{k_{1} r}} p_{0,1} e^{-\beta_{1} r}
$$

and

$$
p_{2}(r)=\sqrt{\frac{2 \theta_{2}^{\text {crit }}}{k_{2} W}} p_{0,2} e^{-\beta_{2} r}
$$

where $\beta_{1}$ and $\beta_{2}$ are the modal attenuation coefficients.
Taking the ratio of these two expressions, with $p_{0,1} \approx p_{0,2}$ and $k_{1} \approx k_{2}$ (just to make interpretation easier), we obtain

$$
R_{2 / 1}=\sqrt{\frac{2 r \theta_{2}^{\text {crit }}}{W}} e^{-\left(\beta_{2}-\beta_{1}\right) r}
$$

We see that the mode 2 ducting "gain" of the $\sqrt{r}$ should be counteracted by the exponential decrease in mode 2 by exponential attenuation. Thus, at "sufficiently far range," the curved ducting effect could be lost, and only the initial beaming and refraction of the low modes by the curved duct would be observed. In terms of angular deflection of the energy, the energy that is ducted by the curved duct over large distances, and thus would show a large angle deflection, would likely be lost due to attenuation. Let us put in some numbers for this. Using a normal mode code to calculate $\beta_{n}$, we see that for $100 \mathrm{~Hz}$ and the parameters used above, $\beta_{2}=4.38 \times 10^{-5} \sim 3 \beta_{1}$, and for $200 \mathrm{~Hz}$, $\beta_{3}=3.93 \times 10^{-5} \sim 2 \beta_{2}$. If we take $W=250 \mathrm{~m}$ and use $\theta^{\text {crit }} \sim 4.7^{\circ}$ for all modes, we obtain that $R_{2 / 1}=0.7$ (the $6-\mathrm{dB}$ down point) is at $780 \mathrm{~m}$ for $100 \mathrm{~Hz}$ and $R_{3 / 2}=0.7$ is at $770 \mathrm{~m}$ for $200 \mathrm{~Hz}$. These are rather modest distances. Moreover, these estimates do not account for the fact that a curved path from the source to a receiver is always longer than a straight line (geodesic) path, which can make the curved path up to a factor of $\pi / 2$ longer for a semicircle. However, on the other hand, it also does not account for the detailed interferences within the duct, which can produce focusing regions of high intensity at ranges greater than one would predict from the preceding sonar equation (average field) type of argument. This discussion points out that the curved ducted path can still be energetically significant compared to a straight line path over a few kilometers, but probably not much more for the size and strength of internal waves seen in SW06. However, stronger $\Delta C$ internal waves and larger amplitude internal waves (which are often seen elsewhere in the world's oceans), could easily make the distances over which curved paths have appreciable ducted energy larger.

We might also look more closely at the energetics of the leaking beams. These modal beams are ducted initially, over about a mode cycle distance, after which they leak out. Thus, they do not spread like the ducted modes up to about $\Delta_{n m}$, and then they spread cylindrically. Thus, past $\Delta_{n m}$, we have

$$
p_{1} \sim p_{01} \sqrt{\frac{1}{p_{1}\left(r-\Delta_{n m}\right)}} e^{-k_{1} r}
$$

which slightly modifies our previous ratio results. We note that the refraction of the beam upon escape from the duct can also tighten (focus) the escaping beam slightly, but this is expected to be a very small effect.

As a final note in this section, before we proceed to the numerical modeling and data results, we discuss what a "more realistic" curved duct model would entail, again with the acoustics in the context of the Weinberg-Burridge formalism. To begin with, one could use a realistic $c(x, y, z)$ field, based on data, numerical models, or a combination of both. From this, one can create a more detailed $n_{n}(\vec{r})$ field, using the exact model eigenvalues, as opposed to our perturbation results. From this, one could find slightly more detailed horizontal model ray trajectories, which certainly have some physical insight, but on a 

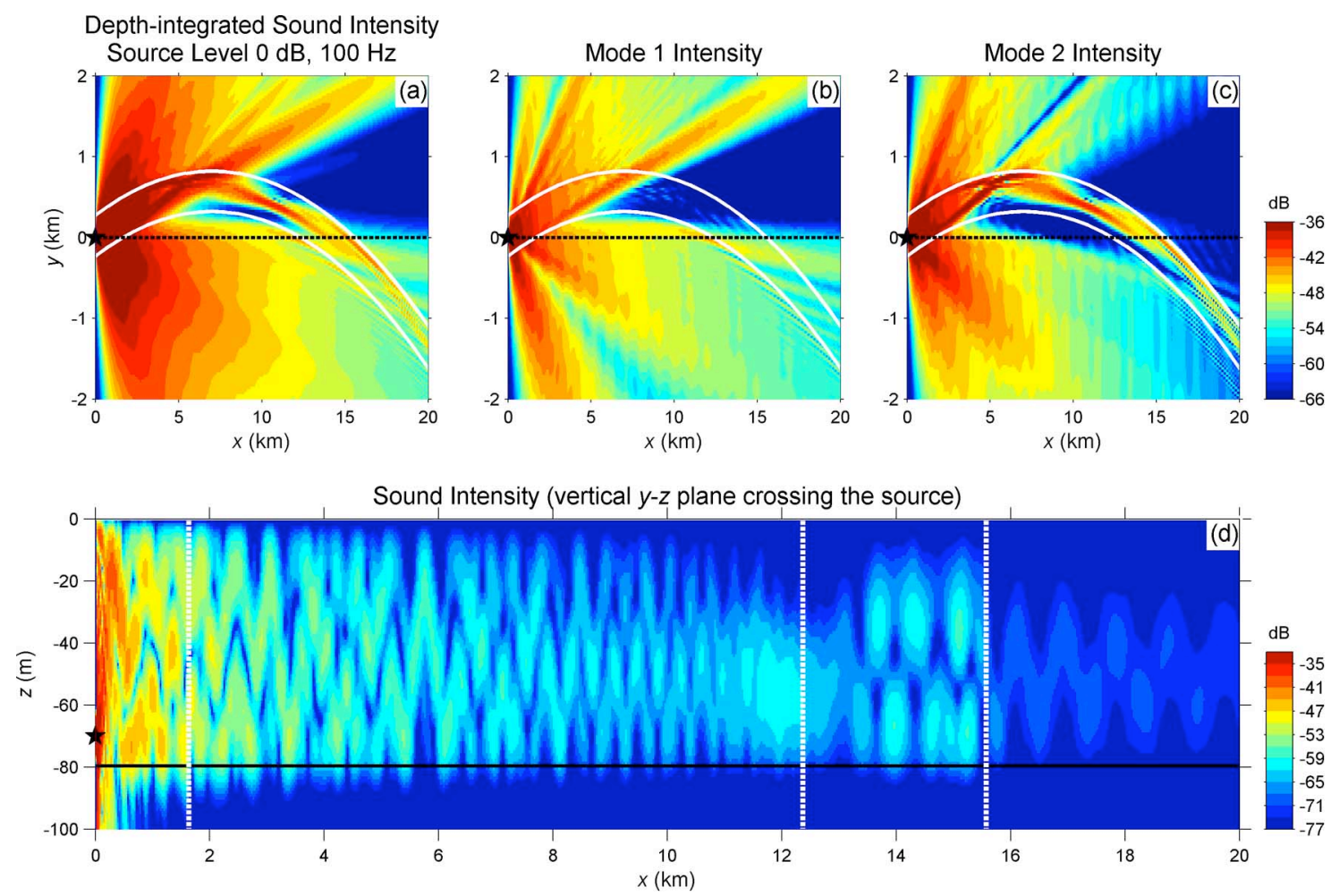

Fig. 11. (a) Depth-integrated sound intensity at $100 \mathrm{~Hz}$. (b) Vertical mode 1 intensity. (c) Vertical mode 2 intensity. (d) Vertical slice of sound intensity at $y=0$ (all dB units are unity source level). Note clear appearance of mode 1 and 2 between ranges 12 and $15 \mathrm{~km}$ (in the internal wave duct). Radius of curvature is 45 $\mathrm{km}$ here.

more visual basis. However, the tradeoff for this greater computational fidelity and accuracy is a loss of transparency of the physics, at least in the analytical form sense. With these comments in mind, it is now appropriate to look at the more detailed numerical model.

We also note that the trajectory of mode coupled beams escaping the waveguide can also be described by the ray-mode calculations, simply by having mode $n m \rightarrow n m^{\prime}$ at the coupling interface and then using the modal index of refraction for $n m^{\prime}$ after coupling. Given close coupling, these coupled mode trajectories will cluster in a beam around the adiabatic angle for $n m$. We also note that use of Snell's law using the local modal index of refraction describes the paths incident on and refracting through the internal wave duct boundaries.

\section{NumeriCAL MODELING}

In this paper, to handle a realistic ocean case, we employed a Cartesian 3-D parabolic equation acoustical propagation program [20], which is briefly described below, and the reader is referred to the report for further details. This program employs the split-step Fourier (SSF) technique [21] to solve the PE for one-way propagating waves from a monotonic source. The SSF technique divides propagation over each distance increment through a heterogeneous sound-speed environment into step-by-step "free space" propagation through a medium having a fixed reference wave number, and periodically introduced (at each step) phase fluctuations consistent with departures from that fixed speed. The free space propagation is handled in the wave number domain, and the phase anomalies are introduced in the spatial domain. Amplitude effects such as absorption are introduced with the phase anomalies. Thus, each step requires a 2-D Fourier transform and an inverse 2-D Fourier transform. Note that the wide angle variant of the propagation operator is used [22].

\section{A. Sound Ducting-Source Inside Internal Wave Train}

Fig. 11 shows images of mode ducting and dispersive refraction "leaking" created by the numerical model that are quite striking, as well as visually easy to interpret. We show two examples here at 100 and $200 \mathrm{~Hz}$. In Fig. 11(a)-(c), we show a plan view of the depth-integrated total energy, the mode 1 energy, and the mode 2 energy, respectively, at $100 \mathrm{~Hz}$. We see both trapping and leakage from the curved duct, as well as the initial escape of higher angle energy near the source. We also see that (filtered) mode 1 is not trapped by the curved waveguide, but leaks out the higher grazing angle side, as expected. However, mode 2 is trapped by the curved duct, in accordance with our theory which predicts that higher modes trap better.

We also see three other effects in Fig. 11(a)-(c). First, the mode 1 energy that escapes the duct is still tightly beamed, with an initial beamwidth on the order of the duct size (though susceptible to cylindrical spreading after that). Second, the trapped mode 1 energy shows distinct focusing/defocusing patterns within the horizontal duct. This is due to the horizontal modal interferences that occur. Finally, the overall curved wave produces some striking focusing (caustic) and shadow zones, which will be discussed later. 


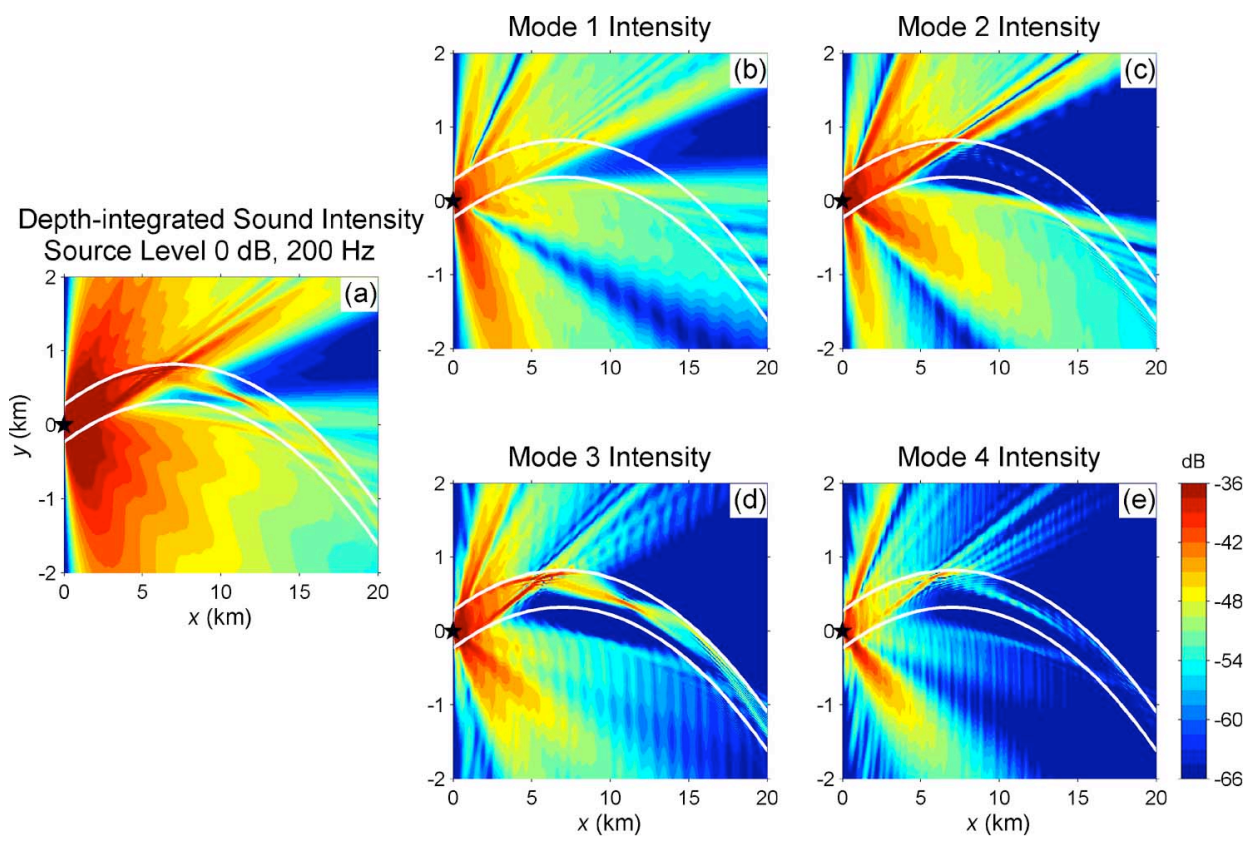

Fig. 12. (a) Depth-integrated sound intensity at $200 \mathrm{~Hz}$. (b)-(e) Vertical modes 1-4, respectively. Curvature of radius is $45 \mathrm{~km}$ here.
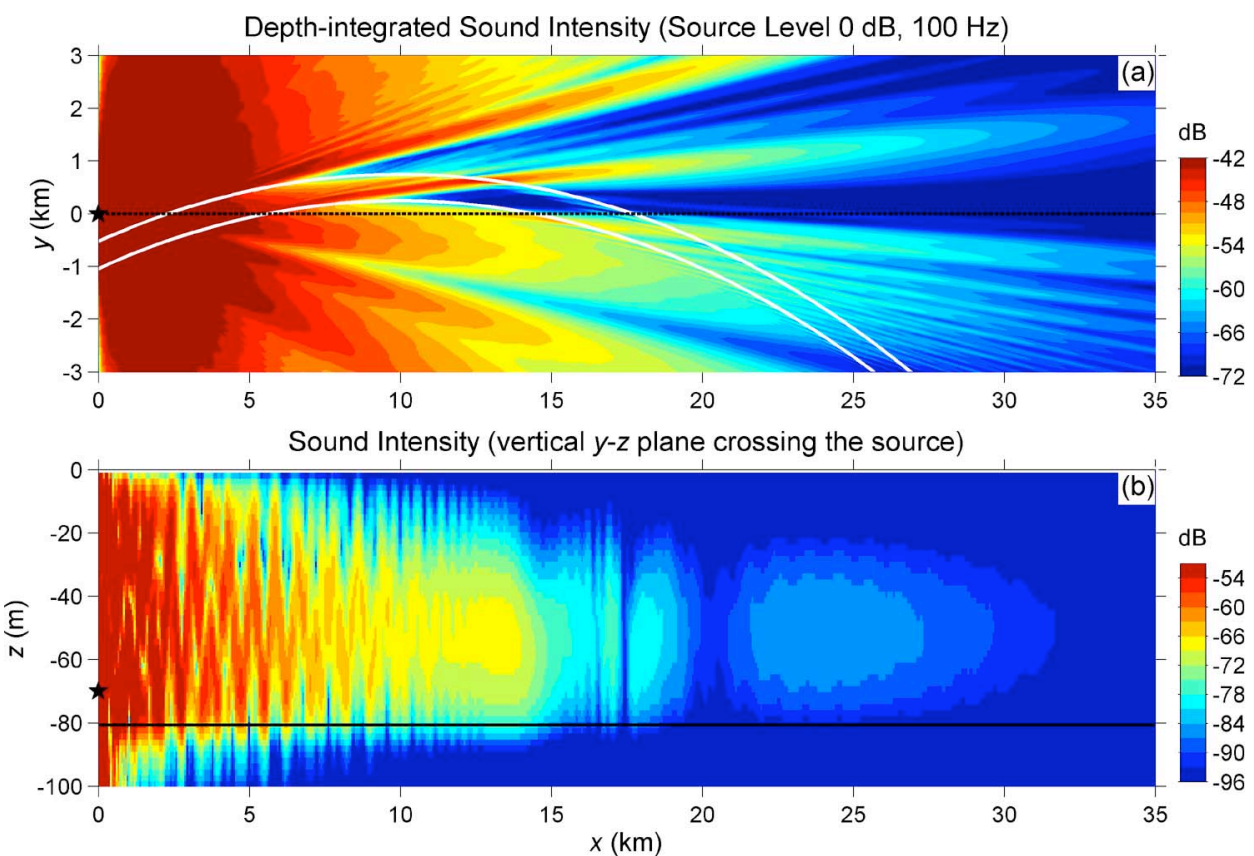

Fig. 13. Shadowing and horizontal Lloyd's mirror effects seen due to a curved nonlinear internal wave of 40-km radius. (a) Depth-integrated sound intensity at $100 \mathrm{~Hz}$. (b) Vertical slice of sound intensity at $y=0$ (all dB units are unity source level).

We next look at a 200-Hz example, in Fig. 12. Fig. 12(a) shows the total depth-integrated energy, whereas Fig. 12(b)-(e) shows filtered modes $1,2,3$, and 4 (only the first four vertically trapped modes are shown). The picture is quite similar to the $100-\mathrm{Hz}$ case, only with more modes. It is seen that modes 1 and 2 are untrapped, whereas modes 3 and 4 are trapped. Moreover, modes 1 and 2 again leak in horizontal beams, and again trapped modes 3 and 4 show focusing/defocusing along the path. One can also see, upon close examination, some slight horizontal refraction of the leaking beams, though this is quite small (fractions of a degree). This is the dispersive "rainbowing" of the leaking energy. The strong acoustic focusing and shadowing regions are again prominent.

\section{B. Shadowing-Source Exterior to a Curved Internal Wave Train}

Numerical examples of this geometry using the waveguide parameters appropriate to SW06, as discussed above, are presented in Figs. 13 and 14. Putting a source just outside curved internal waves of radii 40 and $250 \mathrm{~km}$, one sees very clear shadows being cast from both the exterior and interior walls of the internal wave. Moreover, one sees an interesting horizontal inter- 

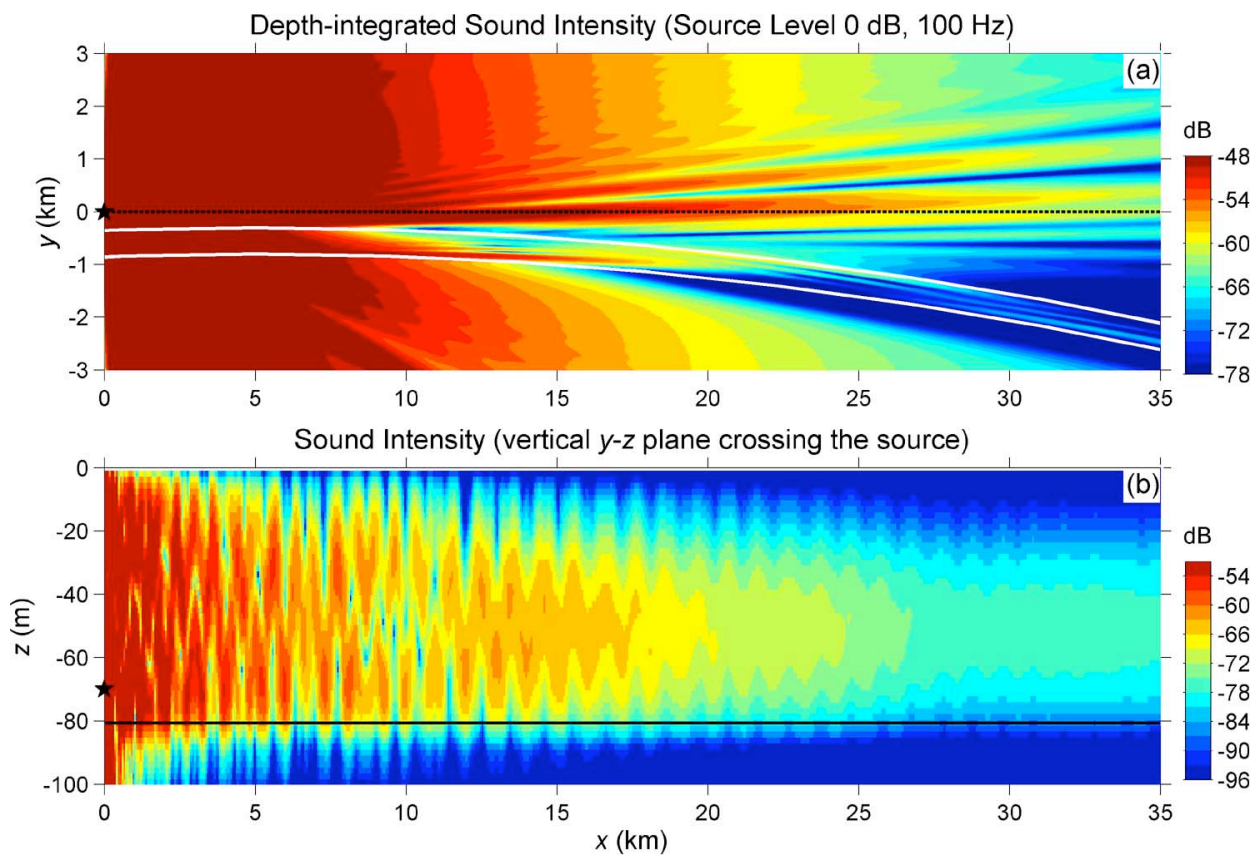

Fig. 14. Shadowing and horizontal Lloyd's mirror effects seen due to a curved nonlinear internal wave of 250 -km radius. (a) Depth-integrated sound intensity at $100 \mathrm{~Hz}$. (b) Vertical slice of sound intensity at $y=0$ (all dB units are unity source level). Note that both shadowing and ducting are seen.
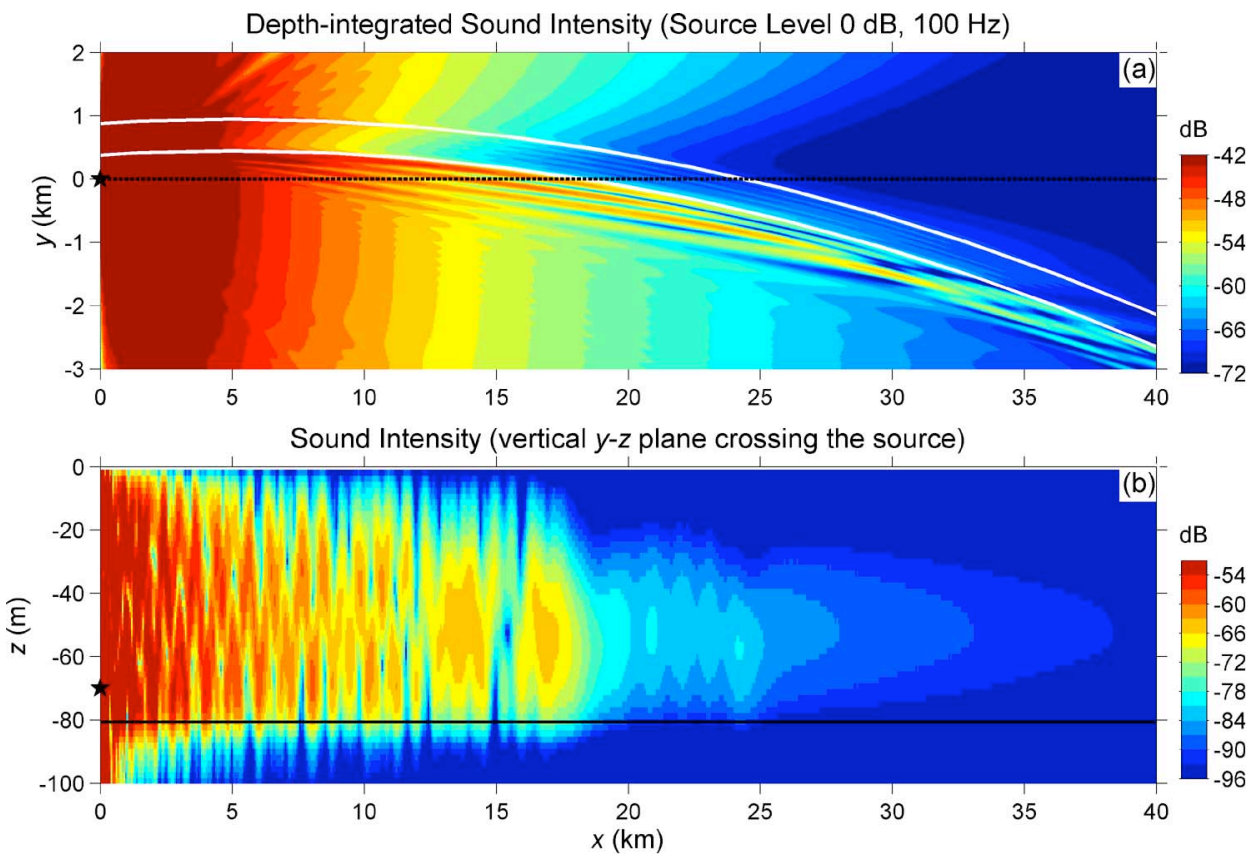

Fig. 15. Whispering gallery, duct penetration, and shadowing effects seen due to a curved nonlinear internal wave of 200-km radius. (a) Depth-integrated sound intensity energy at $100 \mathrm{~Hz}$. (b) Vertical slice of sound intensity at $y=0$ (all $\mathrm{dB}$ units are unity source level).

ference pattern in the upper portion of the figures, due to the interference of the direct and reflected waves, an effect called the "horizontal Lloyd's mirror" [19]. This shadowing of nearby sources might indeed be one of the major causes of amplitude fluctuations seen due to curved nonlinear internal waves. Fig. 14 shows both shadowing and ducting from an outside source.

\section{Source Interior to Curved Internal Wave Train}

In Fig. 15(a) and (b), we see a plan view and a side view, respectively, of the field due to a source placed on the interior of a curved internal wave field $(R=40 \mathrm{~km})$. In the plan view [Fig. 15(a)], most of the acoustic energy through the internal wave duct penetrates directly until 5-km range where it corresponds to very low grazing angles. At this range, the energy which is transmitted through the internal wave shows a slightly scalloped interference pattern due to internal reflections which are positively or negatively interfering depending on the relative path length across the duct.

The energy reflected on the interior side of the curved internal wave forms a whispering gallery effect. Moreover, we see the horizontal Lloyd's mirror effect again, as the direct and whispering gallery waves interfere. We also see a strong shadow zone in between the exterior and interior regions. Fig. 15(b) illustrates these effects in a vertical plane. 

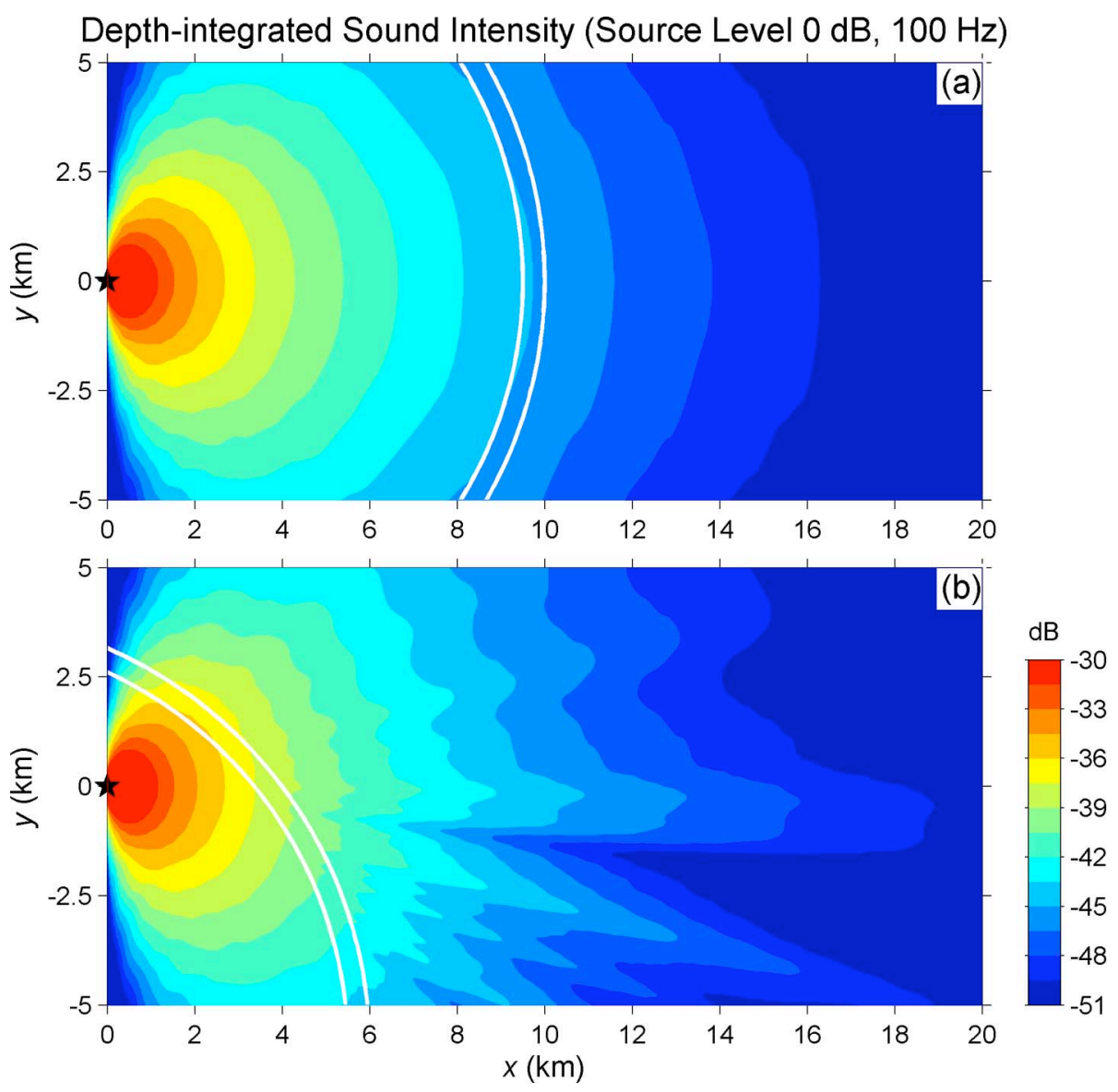

Fig. 16. Source distant from interior to an internal wave duct. (a) Control case showing the source at the center of the curved duct. (b) Energy penetration when the source is not at the center (all $\mathrm{dB}$ units are unity source level). The radius of the curved nonlinear internal wave is $10 \mathrm{~km}$.

Fig. 16(a) and (b) shows a slightly different geometry for the "source interior to the internal wave" with the sound source placed more distant from the highly curved internal wave. In Fig. 16(a), we show a horizontal view of the source at the center of a circular internal wave, which is actually a control case. The sound energy should show circularly symmetric transmissions through the wave, which it does. In Fig. 16(b), we again see the slightly scalloped pattern of energy penetrating the internal wave duct, as before, but no interior whispering gallery rays - this is due to the grazing angles now being too high for subcritical angle total reflection to occur.

\section{SW06 ARrival ANGLes FiEld DATA}

To verify that refractive effects occur when sound is transmitted along paths that are roughly parallel to curved (or straight) packets of nonlinear internal waves, we look at sound transmitted along such a path in SW06. Fig. 1 shows the geometry of this path with respect to a snapshot of wave activity. A source called "The Miami Sound Machine" transmitted 100-, 200-, 400-, 800-, and 1600-Hz phase encoded sequences as part of SW06, and we will consider its transmissions here. In particular, we will analyze the $200-\mathrm{Hz}$ transmissions here, received at a joint horizontal line array (HLA) and vertical line array (VLA). The HLA was oriented close to north/south on the seafloor with the VLA at the southern end. Sound transmitted along this path was analyzed by Collis et al. [23] for horizontal coherence study and further information on the experimental equipment can be found in that paper.

The center of the HLA was $19.2 \mathrm{~km}$ from the source. The bearing from the array center to the source was $26.2^{\circ}$. With this along-shelf path geometry, the major internal wave propagation direction across shelf was very nearly perpendicular to the acoustic track. If mean or time-dependent curvature of acoustic paths occurred and was significant, it would be evident as a deflection of the apparent source angle from $26.2^{\circ}$, as well as in intensity fluctuations. Because our paper focuses mainly on angular deflection, and because this should be a more direct indication of deflection effects than intensity fluctuations, we will look at angular wander using SW06 HLA time series of the heading of maximum array gain. We will then correlate this data against measurements of the internal wave at the receiver site to try to understand the angular fluctuations observed.

Fig. 17 shows both internal wave and acoustics data over a three-day time interval. The internal wave activity in Fig. 17(a) (more fully described below) and the acoustic fluctuations in Fig. 17(b)-(e) are visually correlated. The results presented in the panels will first be described in some detail, then they will be interpreted for ducting and scattering effects.

Fig. 17(a) contains a time series of the highpass filtered depth profile of vertical water velocity measured with an acoustic Doppler current profiler (ADCP) moored approximately midway between the source and the receiver. This time 


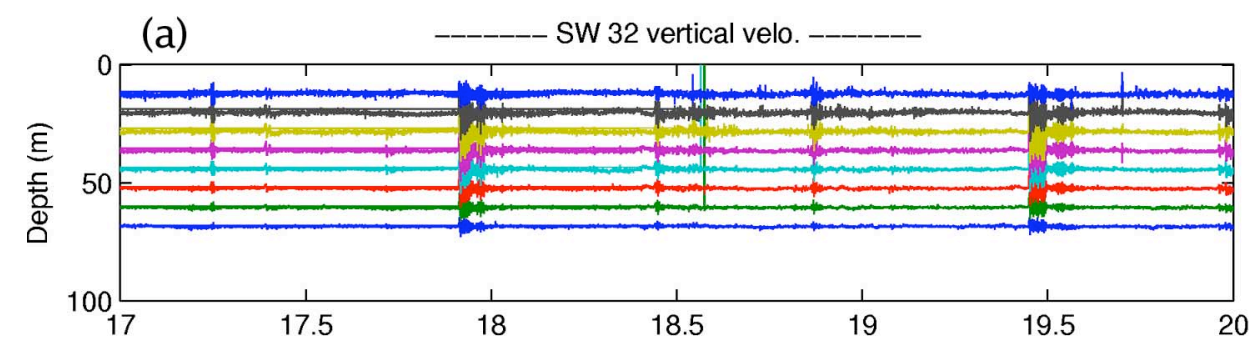

(b)

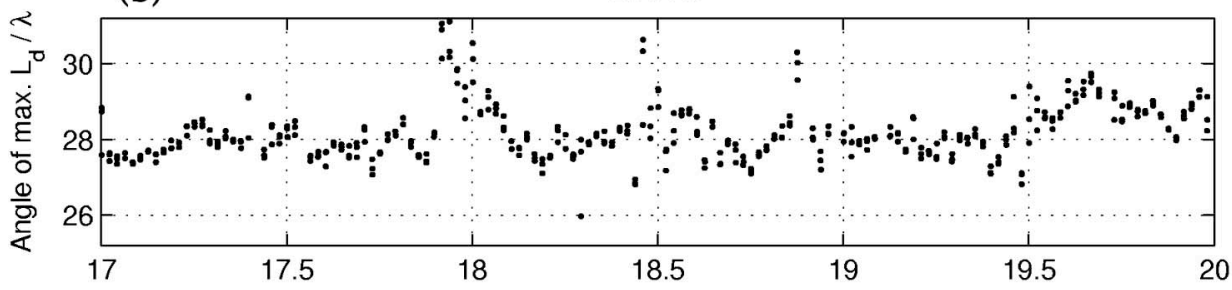

(c) Red: max length. Blue: Fixed angle (28.2 deg) length

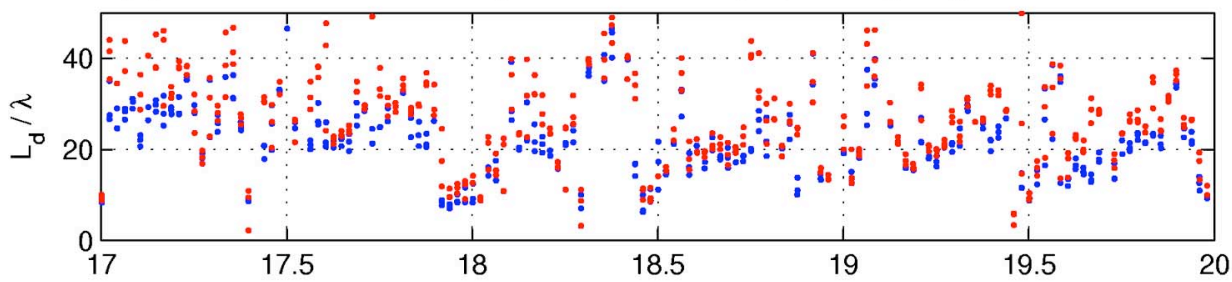

(d)

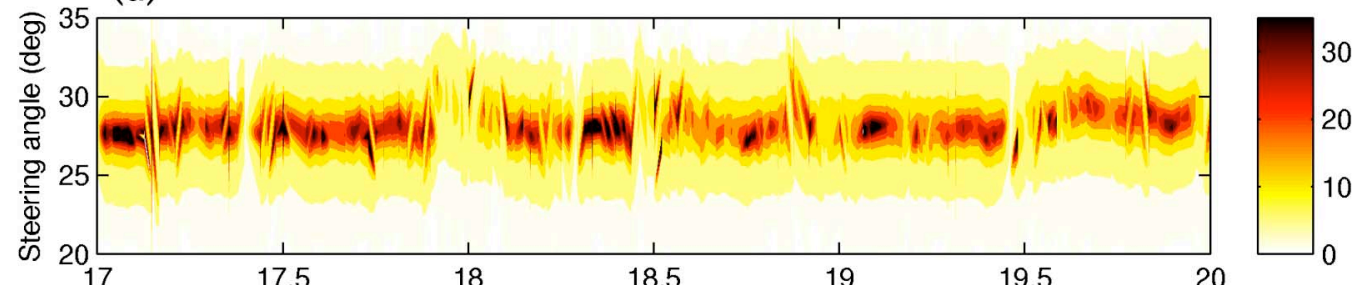

(e)

Mode Power

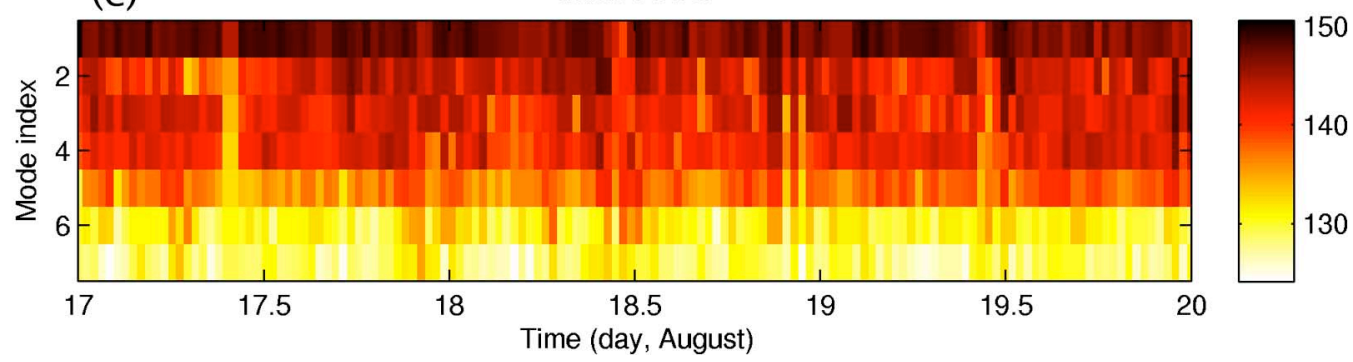

Fig. 17. (a) Highpass filtered vertical velocity time series, indicating times of large internal wave activity. (b) Angle which produces the largest horizontal correlation length of the acoustic signal along the array, a good proxy for angular deflection. (c) Horizontal correlation length along the array compared to the acoustic wavelength. (d) Normalized correlation length/acoustic wavelength. (e) Power in each of the received normal modes (dB re arbitrary reference).

series at representative depth bins clearly shows soliton packet activity, especially on days 18 and 19.5 , which correspond to the spring tide phase of the tidal cycle. Moreover, the spacing between the tides shows a rough (on the average) M2 tidal period of $12.42 \mathrm{~h}$, with some deviation, as noted by Colosi et al. [24]. This time series is rather typical of Mid-Atlantic Bight continental shelf internal wave data.

Fig. 17(d) is the master panel from which the results in Fig. 17(b) and (c) are obtained. The plotted values are the points where the spatially lagged correlation function of the complex matched-filter arrival waveforms falls to the value $e^{-1}$. The computation method is described in [23].
In Fig. 17(b), we see a time series of the steering angle yielding the longest horizontal correlation length along the receiving array (as a function of steering angle) of the arriving acoustic pulses. For each time, these values refer to the angles of maximum correlation length, shown as a function of steering angle in Fig. 17(d). This angle is a good proxy for the angular deflection that we wish to examine. Very interestingly, the angular deflections (from $\sim 27^{\circ}$ to $\sim 31^{\circ}$ ) seen during strong internal wave events are consistent with the $4.7^{\circ}$ critical angle limit one would expect for ducting. Also, we note that on day 19.45 (before noon August 19, 2006), the array was actually moved along the seafloor by an internal wave packet, with the apparent bearing 
to the source changing from $27.5^{\circ}$ to $29^{\circ}$. It is critical that the departure of the array shape from rectilinear be included in the correlation length analysis; the shape changed significantly at the time of the strong wave packet, and a trend was introduced.

In Fig. 17(c), a time series of the maximum observed correlation length is shown, in units of an acoustic wavelength. Two values are shown for each analyzed pulse: the maximum value [from those computed over a wide range of steering angles, shown by color in Fig. 17(d)], and the value looking at one particular steering angle. It is seen that these numbers have an average of about 25 wavelengths, in good agreement with the work of Carey [25]. Also, during periods of strong internal wave activity, this correlation length decreases significantly, in agreement with what was found numerically by Finette and Oba [4] and previously found in the limited analysis of Collis et al. [23]. This lends further credence to the possibility that ducting was one of the mechanisms strongly affecting the acoustic signal.

In Fig. 17(e), we display the power in each of the received normal modes, obtained by mode filtering at the VLA located at the end of the HLA. Mode analysis procedures are described in [23]. This plot shows that internal wave events are generally accompanied by evidence of mode coupling, i.e., some energy is lost by mode 1 and is coupled into the higher order modes. As mode ducting is an adiabatic effect, this shows that some of the energy is crossing the internal wave crests above the critical grazing angle. This is not surprising in that the internal waves seen in SW06, while propagating in the mean approximately perpendicular to the acoustic track, have a directional spectrum that has an angular spread of a few tens of degrees (which includes curvature effects), as shown in Fig. 4. Thus, when the internal wave packets cross between the source and the receiver, the acoustic track can often be at above the critical grazing angle. As an example, a curved wave with diameter less than or equal to the source-receiver distance $R$ is not an unlikely case for our SW06 data, as $R \sim 20 \mathrm{~km}$, and the SAR images show many curved internal wave trains of this diameter or smaller. When such a wave propagates between the source and the receiver, some mode coupling will occur. This situation of concurrent refraction and mode coupling causes complications when applying adiabatic mode methods [26] and coupled mode methods [27].

A scenario begins to emerge from the data and theory, and we now present a "first-order guess" as to what the data indicate. To begin with, as suggested by our theory and the data, it seems unlikely that strongly curved internal waves are ducting energy to the receiver at high angles compared to the bearing to the source. Both the modal energetics (coupling) and the relatively narrow directional spectrum of the internal waves seem contrary to that possibility in SW06. Moreover, large angular deflections are rarely seen in the acoustic data, and are more easily ascribed to mode coupling. (Since our array is not broadside to the source, mode coupling can produce this angular effect because of the vertical/horizontal phase trend ambiguity.) Second, looking at the angular deflection data combined with the correlation length data, it is likely that some ducting is occurring along the path. This duct can be: 1) a straight line duct, 2) a weakly curved duct, or 3) a truncated duct [14] and still produce very similar effects, i.e., wobble of the apparent source direction by up to $\pm \theta_{\text {crit }}$ and large amplitude fluctuations. Third and finally, there
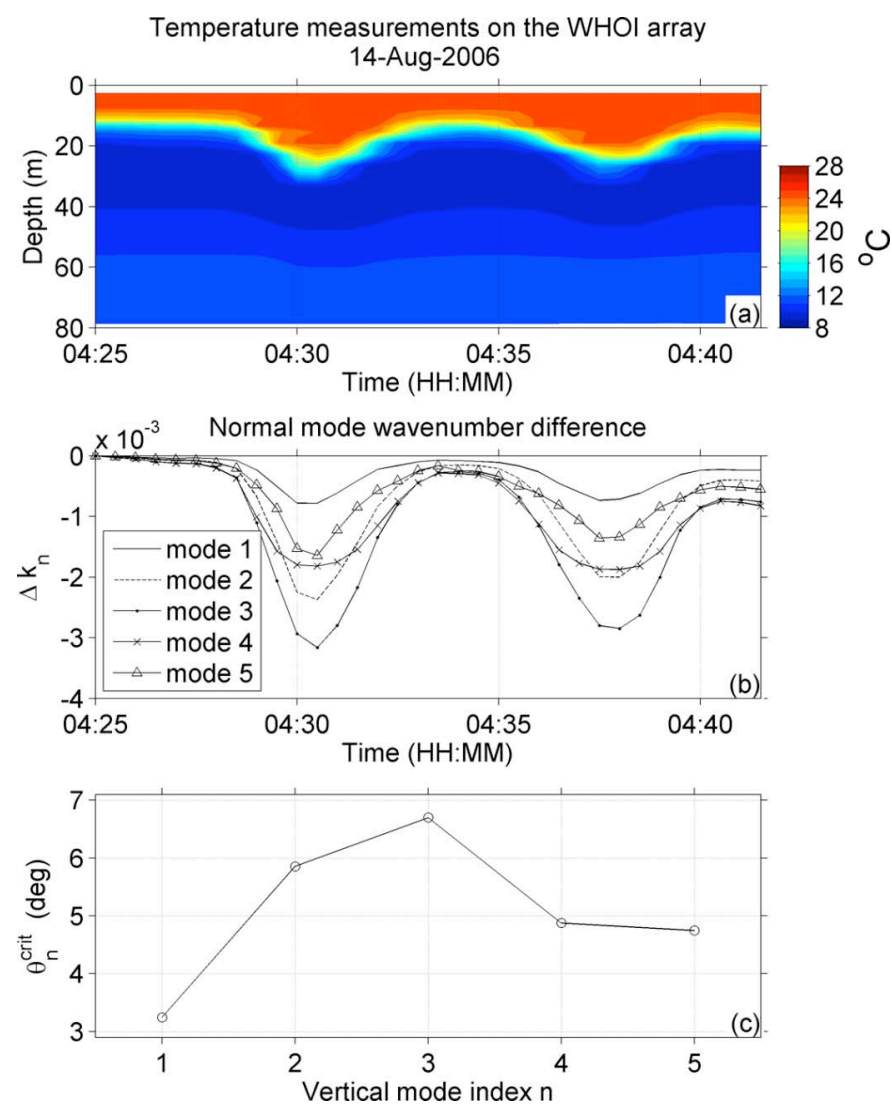

Fig. 18. (a) Internal wave event during SW06. (b) Perturbation of the acoustic mode wave number to the background state at $100 \mathrm{~Hz}$. (c) Critical angle as a function of mode number.

is evidence of time-variable mode coupling in the data, as seen by both the mode amplitude time series and also from the fact that some occasional large angle deflections are seen, which are not due to curved wave ducting (from our previous arguments.) This coupling is likely due to incidence angles upon the internal waves above critical grazing angle, which is supported by the directional spectrum spread of the internal waves.

This scenario is consistent with our data, but we will not claim that it is conclusive proof, as there could be other explanations for the data (though we do not readily see such others.) We think that combining a directional spectrum of nonlinear internal waves, including curved and truncated waves, which induces both ducting and coupling effects, can produce a rather large variety of data effects and that the SW06 data set is just a beginning look at this rich area.

Before leaving the data analysis section, we will consider one more aspect of the SW06 experimental data which is relevant to this analysis, namely, the oceanographic data for the water column and bottom sound-speed profiles. These data show a large variation in the sizes, shapes, and strengths of the nonlinear internal waves, and also show that the bottom is significantly penetrable to sound. These two effects will change the background normal modes from what was used in Section III, as well as the perturbations of those modes by the internal waves. To show how a more realistic profile affects the critical angle calculations presented in (10), we look at a "typical" internal wave duct from SW06 [Fig. 18(a)], and calculate the modes at 
$100 \mathrm{~Hz}$, their eigenvalue perturbations from the background profile, and the critical angle versus mode number that one sees using the realistic profiles.

Doing this calculation, one obtains the results seen in Fig. 18(b) and (c). In Fig. 18(b), we show the eigenvalue perturbation as a function of time due to a real internal wave. We see that the perturbation time series follows the wave transit for each mode, as expected. More interesting is the critical angle for each mode, shown in Fig. 18(c), which varies from 3.2 to $6.8^{\circ}$. This is somewhat more variability in angle than seen in our "canonical waveguide" example, though the average number is about the same. Also, we see that the critical angle increases for the first three modes, but then decreases and flattens out (to about $4.8^{\circ}$ ) for modes 4 and 5 . This is likely due to these higher modes most strongly feeling the effect of the penetrable bottom, which the low modes do not.

Thus, we see that for an exact description of the ducting effects, the more realistic ocean and bottom models should be considered. Also, this last calculation used a "typical" nonlinear internal wave from SW06, but it is well known that the internal waves vary even within a wave train, as well as with space and time. The bottom also varies spatially. Thus, it would not be amiss to eventually consider a more statistical look at the ducting behavior of the modes in shallow-water internal waves. This will be pursued in a future paper.

\section{CONClusion AND Future DiRECTIONS}

In concluding this paper, we will look at the three viewpoints that we examined the ducting effects from, i.e., theory, numerical models, and experimental data. We will briefly state what we have accomplished, but more importantly, examine what yet needs to be done to fully understand curved wave ducting phenomena. This is a rich area, and has more depth than one paper could cover.

Regarding theory, we have endeavored to show, in the context of simple perturbation theory and a canonical ocean model, how the physics of trapping and leaking of acoustic energy from curved nonlinear internal waves works. In terms of things that still need more work, we would point to two items. First, there are the "whispering gallery" modes, in which the sound interacts only with the exterior walls. Second, there is theory that should be developed for full wave trains, not just the "single duct" that we considered in the paper. Wave trains, which have (for instance) rank ordering in size, can show additional trapping and leaking effects in addition to the (basic) case that we showed in this paper.

In terms of numerical models, we have been able to show rather clearly the acoustic effects of single ducts, to match the theory we presented here. However, the models can handle more complex environments, and that is where we should be going with them. In addition to wave trains (as mentioned above), we should also be looking at realistic 3-D internal wave fields based on data. This would allow us to get at the scattering statistics (for intensity, arrival angle, etc.) that we will see in the data.

As to data, the SW06 data were able to give us a very good look at the oceanography of the curved internal waves, so that we can create very good input for the acoustics calculations. The acoustics data, while unique and showing some of the effects we discussed in this paper, were not adequate to unam- biguously describe all of the effects we discussed. This is for the simple reason that the experiment was not designed completely around the goals of this paper: the horizontal/vertical array had many other purposes to accomplish. Thus, we did not have the array completely broadside to the acoustic path (which would have made the angular deflection signal unambiguous, even in the presence of mode coupling), nor did we concentrate all our oceanography measurements along the acoustic track we discussed. We also could have placed point receivers to look at shadowing effects, etc. These are things that we might consider doing in future experiments.

\section{ACKNOWLEDGMENT}

The authors would like to thank H. Graber and N. Williams for providing them with the SAR satellite images they used for this study, and G. Gawarkiewicz for the coastal oceanography input to this paper. They would also like to thank the WHOI SW06 deployment/recovery team, along with the captains and crews of the $R / V$ Knorr, the $R / V$ Oceanus, and the $R / V$ Endeavor.

\section{REFERENCES}

[1] J. Zhou, X. Zhang, and P. H. Rogers, "Resonant interaction of sound wave with internal solitons in the coastal zone," J. Acoust. Soc. Amer., vol. 90, pp. 2042-2054, 1991.

[2] J. R. Apel, M. Badiey, C.-S. Chiu, S. Finette, R. Headrick, J. Kemp, J. F. Lynch, A. Newhall, M. H. Orr, B. H. Pasewark, D. Tielbuerger, A. Turgut, K. von der Heydt, and S. Wolf, "An overview of the 1995 SWARM shallow-water internal wave acoustic scattering experiment," IEEE J. Ocean. Eng., vol. 22, no. 3, pp. 465-500, Jul. 1997.

[3] M. Badiey, Y. Mu, J. F. Lynch, J. R. Apel, and S. N. Wolf, "Temporal and azimuthal dependence of sound propagation in shallow water with internal waves," IEEE J. Ocean. Eng., vol. 27, no. 1, pp. 117-129, Jan. 2002.

[4] S. Finette and R. Oba, "Horizontal array beamforming in an azimuthally anisotropic internal wave field," J. Acoust. Soc. Amer., vol. 114, pp. 131-131, 2003.

[5] B. G. Katznelson and S. A. Pereselkov, "Low-frequency horizontal acoustic refraction caused by internal wave solitons in a shallow sea," Acoust. Phys., vol. 46, pp. 684-691, 2000.

[6] D. J. Tang, J. F. Lynch, J. N. Moum, P. Abbot, N. R. Chapman, P. H. Dahl, T. F. Duda, G. G. Gawarkiewicz, S. Glenn, J. A. Goff, H. Graber, J. N. Kemp, A. R. Maffei, J. D. Nash, and A. E. Newhall, "Shallow Water '06: A joint acoustic propagation/nonlinear internal wave physics experiment," Oceanography, vol. 20, no. 4, pp. 156-167, Dec. 2007.

[7] R. Burridge and H. Weinberg, "Horizontal rays and vertical modes," in Wave Propagation and Underwater Acoustics, J. B. Keller and J. S. Papadakis, Eds. Berlin, German: Springer-Verlag, 1977, pp. 86-152.

[8] T. F. Duda, J. F. Lynch, Y. T. Lin, A. E. Newhall, H. Graber, and M. Caruso, "The effects of non-linear internal wave curvature on acoustic propagation," J. Acoust. Soc. Amer., vol. 123, p. 3588, 2008.

[9] J. D. Irish, J. F. Lynch, J. N. Kemp, T. D. Duda, and A. E. Newhall, "A moored array for measuring internal solitary waves during Shallow Water'06," in Proc. IEEE/MTS OCEANS Conf., Vancouver, BC, Canada, Oct. 2007, DOI: 10.1109/OCEANS.2007.4449170.

[10] C. C. Boughan and W. Siegmann, Private Communication 2008.

[11] Y. Matsumura and H. Hasumi, "A non-hydrostatic ocean model with a scalable multigrid Poisson solver," Ocean Model., vol. 24, pp. 15-28, 2008.

[12] C. Y. Shen, T. E. Evans, R. M. Oba, and S. Finette, "Three dimensional hindcast simulation of internal soliton propagation in the Asian Seas International Acoustics Experiment area," J. Geophys. Res., vol. 114, no. C1, p. C01014, 2009.

[13] J. F. Lynch, T. F. Duda, Y. T. Lin, A. E. Newhall, and P. F. J. Lermusiaux, "A model for four-dimensional coastal internal waves with applications to acoustics," J. Acoust. Soc. Amer., vol. 126, p. 2305, Oct. 2009.

[14] Y. T. Lin, T. F. Duda, and J. F. Lynch, "Acoustic mode radiation from the termination of a nonlinear internal gravity wave duct in a shallow ocean area," J. Acoust. Soc. Amer., vol. 126, no. 4, pp. 1752-1765, Oct. 2009 . 
[15] T. F. Duda, "Acoustic mode coupling by nonlinear internal wave packets in a shelfbreak front area," IEEE J. Ocean. Eng., vol. 29, no. 1, pp. 118-125, Jan. 2004.

[16] T. F. Duda and J. Preisig, "A modeling study of acoustic propagation through shallow-water solitary wave packets," IEEE J. Ocean. Eng., vol. 24, no. 1, pp. 16-32, Jan. 1999.

[17] B. G. Katsnelson and V. G. Petnikov, Shallow-Water Acoustics, ser. Books in Geophysical Sciences. New York: Springer-Verlag, 2002, ch. 3 .

[18] E. Hecht and A. Zajac, Optics. Reading, MA: Addison-Wesley, 1976, ch. 5.

[19] J. F. Lynch, J. Colosi, G. Gawarkiewicz, M. Badiey, B. Katznelson, J. Miller, W. Siegmann, C. Chiu, and A. Newhall, "Consideration of finescale coastal oceanography and 3-D acoustics effects for the ESME sound exposure model," IEEE J. Ocean. Eng., vol. 31, no. 1, pp. 33-48, Jan. 2006.

[20] T. F. Duda, "Initial results from a Cartesian three-dimensional parabolic equation acoustical propagation code," Woods Hole Oceanogr. Inst., Woods Hole, MA, Tech. Rep. WHOI-2006-041, 2006.

[21] R. H. Hardin and F. D. Tappert, "Applications of the split-step Fourier method to the numerical solution of nonlinear and variable coefficient wave equations," SIAM Rev., vol. 15, pp. 423-423, 1973.

[22] D. J. Thomson and N. R. Chapman, "A wide-angle split-step algorithm for the parabolic equation," J. Acoust. Soc. Amer., vol. 74, pp. 1848-1854, 1983.

[23] J. M. Collis, T. F. Duda, J. F. Lynch, and H. A. Deferrari, "Observed limiting cases of horizontal field coherence and array performance in a time-varying internal wavefield," J. Acoust. Soc. Amer., vol. 124, pp. EL97-EL103, 2008.

[24] J. A. Colosi, J. F. Lynch, R. C. Beardsley, G. Gawarkiewicz, C.-S. Chiu, and A. Scotti, "Observations of nonlinear internal waves on the outer New England continental shelf during the summer Shelfbreak PRIMER,” J. Geophys. Res., vol. 106, pp. 9587-9601, 2001.

[25] W. Carey, "The determination of signal coherence length based on signal coherence and gain measurements in deep and shallow water," J. Acoust. Soc. Amer., vol. 104, pp. 831-837, 1998.

[26] T. F. Duda, "Examining the validity of approximations to fully three-dimensional shallow-water acoustic propagation through nonlinear gravity waves," in Proc. OCEANS Conf., 2007, DOI: 10.1109/OCEANSE.2007.4302333.

[27] J. C. Preisig and T. F. Duda, "Coupled acoustic mode propagation through continental shelf internal solitary waves," IEEE J. Ocean. Eng., vol. 22, no. 2, pp. 256-269, Apr. 1997.

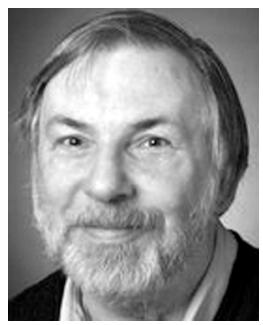

James F. Lynch (M'96-SM'02-F'05) was born in Jersey City, NJ, on June 3, 1950. He received the B.S. degree in physics from Stevens Institute of Technology, Hoboken, NJ, in 1972 and the Ph.D. degree in physics from the University of Texas at Austin, Austin, in 1978.

He then worked for three years at the Applied Research Laboratories, University of Texas at Austin (ARL/UT) from 1978 to 1981, after which he joined the scientific staff at the Woods Hole Oceanographic Institution (WHOI), Woods Hole, MA. He has worked at WHOI since then, and currently holds the position of Senior Scientist in the Applied Ocean Physics and Engineering Department. His research specialty areas are ocean acoustics and acoustical oceanography, but he also greatly enjoys occasional forays into physical oceanography, marine geology, and marine biology.

Dr. Lynch is a Fellow of the Acoustical Society of America, the former Editor-in-Chief of the IEEE JOURNAL OF OCEANIC ENGINEERING and recent chairman of the Applied Ocean Physics and Engineering Department at WHOI.

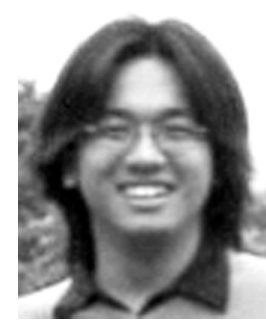

Ying-Tsong Lin (M'10) received the B.S. degree in hydraulic and ocean engineering from National Cheng Kung University, Tainan, Taiwan, in 1996 and the M.S. degree in naval architecture and ocean engineering and the Ph.D. degree in engineering science and ocean engineering from National Taiwan University (NTU), Taipei, Taiwan, in 1998 and 2004, respectively.

From 2002 to 2003, he was a Guest Investigator at the Applied Ocean Physics \& Engineering (AOP\&E) Department, Woods Hole Oceanographic Institution (WHOI), Woods Hole, MA. After receiving his Ph.D. degree, he began a halfyear postdoctoral appointment at Underwater Acoustics Laboratory, NTU, after which he worked at WHOI as a Postdoctoral Researcher. He currently holds the position of Assistant Scientist at the AOP\&E Department, WHOI. His research interests include shallow-water acoustic propagation, acoustical oceanography, geoacoustic inversion, and underwater sound source localization.

Dr. Lin is a member of the IEEE Oceanic Engineering Society and the Acoustical Society of America.

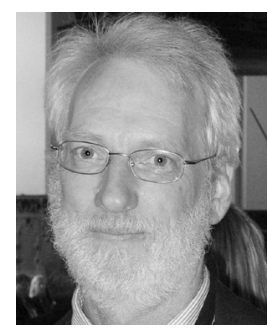

Timothy F. Duda (M'05-SM'09) received the B.A. degree in physics from Pomona College, Claremont, $\mathrm{CA}$, in 1979 and the Ph.D. degree in oceanography from the Scripps Institution of Oceanography, University of California, San Diego, in 1986.

$\mathrm{He}$ worked at the University of California, Santa Cruz, from 1986 to 1991. He has been a Scientist at the Woods Hole Oceanographic Institution (WHOI), Woods Hole, MA, since 1991. His three primary fields of study are ocean acoustic propagation, ocean internal gravity waves, and ocean mixing processes. His research has included physical process studies, development of new measurement tools, and computational acoustic modeling.

Dr. Duda is a member of the IEEE Oceanic Engineering Society. He is also a member of the American Meteorological Society, the American Geophysical Union, and the Acoustical Society of America.

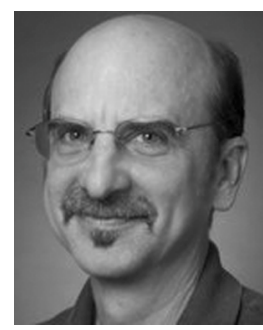

Arthur E. Newhall (M'07) received the B.S. degree in mathematics from the University of Maine at Orono, Orono, in 1985.

$\mathrm{He}$ is a Research Specialist at the Applied Ocean Physics and Engineering Department, Woods Hole Oceanographic Institution (WHOI), Woods Hole, MA. His current interests include ocean acoustic propagation modeling, acoustical oceanography, software engineering, and music.

Mr. Newhall is a member of the IEEE Oceanic Engineering Society and the Acoustical Society of 